\title{
Impedance Adaptive Controller for a Prototype of a Whiplash Syndrome Rehabilitation Device
}

\author{
Isai Guzman-Victoria, ${ }^{1}$ Ivan Salgado-Ramos, ${ }^{2}$ Manuel Mera-Hernandez, ${ }^{1,3}$ \\ Isaac Chairez, ${ }^{1,4}$ and Hafiz Ahmed $\oplus^{5}$ \\ ${ }^{1}$ Unidad Profesional Interdisciplinaría de Biotecnología, Instituto Politécnico Nacional, Mexico \\ ${ }^{2}$ Centro de Investigación y Desarrollo de Tecnología en Cómputo, Instituto Politécnico Nacional, Mexico \\ ${ }^{3}$ Escuela Superior de Ingeniería Mecánica y Eléctrica Ticoman, Instituto Politécnico Nacional, Mexico \\ ${ }^{4}$ Escuela de Ingenieria, Instituto Tecnológico de Estudios Superior de Monterrey, Campus Guadalajara, Mexico \\ ${ }^{5}$ Coventry University, Priory Street, Coventry CV1 5FB, UK
}

Correspondence should be addressed to Hafiz Ahmed; ac7126@coventry.ac.uk

Received 15 January 2019; Revised 2 April 2019; Accepted 28 April 2019; Published 28 May 2019

Academic Editor: Asier Ibeas

Copyright (C) 2019 Isai Guzman-Victoria et al. This is an open access article distributed under the Creative Commons Attribution License, which permits unrestricted use, distribution, and reproduction in any medium, provided the original work is properly cited.

The objective of this study was to design of an output based impedance adaptive controller for a special class of cervical orthoses, a class of biomedical devices for the rehabilitation of neck illnesses. The controller used the adaptive sliding mode theory to enforce the tracking of the reference trajectory if the patient was not resistant to the therapy. If the patient rejects the orthosis activity, a second impedance-based controller governs the orthosis movement allowing the patient to take the leading role in the orthosis sequence of movements. The proposed controller considers a weighted controller combining the tracking and the impedance controls in a single structure. The monitoring of the external force was evaluated on a novel weighting function defining on-line the role of each controller. The proposed orthosis was motivated by the prevalence of whiplash, which is a syndrome that is produced by forced hyperextension and hyperflexion of the neck. This study included the development of a technological prototype of the orthotic type to support the recovery of patients diagnosed with whiplash. The sections that make up the orthotic device are two independent systems that move the patient's head in the sagittal and frontal planes. For this purpose, the mechanical structure of the cervical orthosis was made up of 7 pieces printed in 3D with polylactic acid (PLA). The operation of the cervical orthosis was evaluated in two sections: (a) using a simulation system, which consists of a spring with an artificial head and the development of a graphic interface in Matlab, and (b) evaluating the controller on the proposed orthosis. With these elements, the follow-up of the trajectory proposed by the actuators was evaluated, as well as its performance in the face of the opposition that a patient generates. The superiority of the proposed controller was confirmed by comparing the tracking efficiency with proportional-integral-derivative and first-order sliding variants.

\section{Introduction}

The increasing number of injuries affecting all the articulations in human body has motivated the development of remarkable assistance rehabilitation devices [1]. Exoskeletons, lower and upper limbs robotic orthosis, and so on appear as new medical tools to help the rehabilitation medical doctor and physiotherapists in performing more efficient therapeutic procedures [2]. The methodological and technological approaches aimed at developing such class of rehabilitation devices are now mature and they have produced medical devices which are now validated. Among the illnesses restricting the articulations movements, there are several sicknesses which may affect the mobility of the cervical vertebra. Vertebral herniated disk, radiculopathy, meningitis, and vertebral fractures are the most common pathologies associated with the cervical section of the spinal cord [3-5]. In general, their symptoms include persistent 
pain, controlled mobilization difficulties of the affected zone, and restricted articulation displacements as well as muscular inflammation [6].

The whiplash syndrome is becoming one of the most prevalent cervical pathologies. This syndrome is the consequence of a fast angular movement in either lateral or frontal anatomical planes $[7,8]$. It is recognized as an incapacitate pathology with acute periods of pain and restricted mobilization of the head. This syndrome has been associated with violent automobile collisions [9]. The hyperextension of the muscles and ligaments in the cervical section are the physiological fundamentals of the syndrome. The relevance of this pathology has motivated the introduction of the so-called Quebec classification, which contains the clinical manifestations and the pathology degree. This classification also indicates the suggested treatment for each degree of the pathology [10].

In the last few years, some technological options appeared as alternative technologies for the treatment of neck vertebral illnesses, including the neck hyperextension and hyperflexion (whiplash syndrome) [11, 12]. Most of these devices may ensure the complete immobilization of the vertebral articulation in the cervical area $[13,14]$. The conventional treatment consists of immobilizing the cervical region of the spinal cord by wearing therapeutic rigid collars as well as pharmaceutical therapy [15]. A cervical collar is an orthosis fitting the patient's neck anywhere from the jaw to the chest. It has two major functions: restricting the cervical movements (flexion, extension, and lateral tilt) and supporting the head to allow healing the muscles and the ligaments. In addition, the collar decreases the muscle spasms and control the pain in the neck $[16,17]$.

The rigid or semirigid collars (made of soft material) represent the main assistance technology for the cervical injuries treatment. Nevertheless, there are some drawbacks in using these collars [18]. In particular, the continuous immobilization in all planes can lead to sacral pressure points, heel and elbow breakdown, risk of deep venous thrombosis, muscle atrophy, etc. [19]. Most of these inconveniences can be saved if a complementary mobilization therapy of isometric exercises is realized. It is usual that this complementary therapy is not recommended because the necessity of a qualified physiotherapist intervention [20].

Nowadays, the emerging of rehabilitation technology including the development of active orthosis has motivated the introduction of automatized devices aimed to mobilize the cervical region of patients wearing the rigid collar. The multi-cervical unit is a commercial product (BTE Technologies) aimed at offering pain reduction by restricting the neck lateral movements; however, this device could induce a vertical continuous force which may contribute to the treatment of conical and acute pain, vertebral disks compression, and some others. This equipment requires the recurrent visits of the patient to the specialized clinical facilities [21].

ReSolve Halo is a cervical device with external fixation system. It has the mechanical sections to immobilize the cervical articulations and it offers complementary regulated support for the head. The position of the neck area is fixed in advance and the patient is required to visit the physician if some adjustment is needed. This device offers the weight discharge of the head by fixing the device to the thorax area [22]. The Cervical Thoracic Orthosis provides a conservative treatment for the cervical pathology. It can restrict the rotational movement of the neck, but it can hold the head section immobilized. This device is attached to the patient's thorax to offer the weight discharge [23].

All the devices proposed for aiding the therapeutics of the neck illnesses have the disadvantage of forcing the neck's immobilization, which is not the best solution for the requested rehabilitation. Notice that the mobilization realized by these devices did not consider the potential resistance of the patient to the orthosis activity. This aspect must be taken into account considering the possible pain induced for the application of orthosis-based treatment [24]. The main motivation for developing an active orthosis for the whiplash syndrome is the increasing number of neck illnesses which are consequences of car accidents, long periods of muscle inactivity yielding cervical vertebral inflammations, etc. These sicknesses are treated by fixed orthoses which are supporting the neck by immobilizing the cervical section, but not offering an integral rehabilitation. The proposed orthosis makes an enhancement in this direction because it is mobilizing the neck section in a controlled way, while the patient's head is still held by the orthosis [25-29].

It is usual that active orthosis (mechanized rehabilitation devices with automatized movements) should be controlled by robust controllers which are not considering the patient resistance. This design requirement implies the application of a class of human-robot (or patient-orthosis in this case) interaction framework solution. In such class of approaches, the tracking of reference trajectories still plays a relevant role in the design of the automatic controller. However, the safe robot operation, considering the patient resistance to the orthosis action, plays a primary role because the necessity of ensuring a comfortable assisted rehabilitation [30].

The current control designs to solve the human-robot interaction may not provide sufficient flexibility for collaboration tasks (implying a smooth transition between the tracking and the resistance operation conditions), requiring that only two cases are admissible: the robot leads or follows the human by assessing the performance of human continuously [31]. Only a few approaches have considered the possibility of transiting from the robot leading (orthosis-in-charge) scenario to the human ruling case (patient-in-charge) [32]. Within these works, diverse studies have provided nonlinear approaches to regulate the human-robot interaction [3335] inspired by the hybrid control designs. Within the hybrid solutions, the application of approximate mathematical descriptions to the orthosis model has been used along the last years [36-38]. Such approaches try to produce soft interaction between patient and orthosis. Collaborative robot control is also providing new paradigms to control active orthosis, where the patient exerts the rehabilitation procedure but keeping a predefined safety zone [39-41].

Impedance/admittance-based controllers can solve each of the proposed scenarios independently [42]. However, there is a necessity of changing the desired impedance in each case. This condition may lead to the introduction of 
switched controllers inducing high-frequency oscillations that are not justifiable for the rehabilitation of the cervical region by the proposed active orthosis $[43,44]$. In addition, the common impedance controllers assume that there is an accurate mathematical representation of the robotic device, which is a strong assumption in many realistic cases. Indeed, there is a tread-off between the softness of the automatic control realization ensuring the safe operation of the orthosis (usually solved by adaptive variants of the controller) and the stipulated robustness against the nonmodeled sections of the robotic orthosis. Several options of adaptive controllers have dealt with these design problems such as [45-47], where relevant approaches handle the thread-off between the position and the force based forms.

A major opportunity to solve the trade-off in the cervical orthosis development is the implementation of the so-called adaptive sliding mode theory (ASMT) [48]. The recent advances in the ASMT have led to finding a remarkable design strategy that maintains the finite-time convergence and robustness of the regular sliding mode based controllers but ensures the reduction of the chattering phenomenon (high-frequency oscillations occurring when the sliding mode is enforced). These combined characteristics motivate the application of ASMT as a potential solution to develop impedance adaptive sliding mode controllers to regulate the safe operation of a cervical orthosis. This novel controller represents the major contribution of this study.

The paper is organized as follows: Section 2 details the cervical orthosis proposed for the treatment of the whiplash syndrome. Section 3 defines the impedance adaptive sliding mode controller including the details of the variants of state and output based approach. Section 4 describes the real cervical orthosis developed for testing the proposed adaptive controller. Section 5 describes the numerical evaluation of the proposed controller over a simulated version of the orthosis. Section 6 explains the experimental evaluation of both the developed cervical orthosis and the application of the impedance adaptive controller implemented in an embedded device. Section 7 closes the paper with some final remarks.

\section{The Cervical Orthosis}

The orthosis for the active treatment of the neck disorder associated with the whiplash syndrome considered a fully actuated robotic manipulator. The design considered a fixed body mobilized by two independent articulations supporting the central orthosis body. The robotic system included a supportive device allowing the patient to carry the orthosis without the necessity of an external support. This design agrees with the regular mechanism used to track and move the articulated section of the injured necks of the selected patients.

The cervical active orthosis consisted of a low-weight two-degree-of-freedom robotic manipulator with a special end effector, which can hold the patient's head. The movements induced by the suggested robotic manipulator correspond to the usual mobilization of the cervical region in the rehabilitation therapies. The orthosis was designed considering these main aspects.

The dimensions of the mechanical orthosis design were not prefixed. Indeed, the orthosis design allowed its configurations modifications corresponding to averaged anthropomorphic measures. A general view of the computer assisted design version of the proposed rehabilitation device appears in Figure 1.

Based on the mechanical structure associated with the neck orthosis, let us consider that $q \in \mathbb{R}^{2}$ describes the joints configuration, while $P \in \mathbb{R}^{3}$ describes the position of the center section in the upper part of the orthosis (Figure 1) in the task space.

The position of point $P$ is related to the join configuration by the nonlinear function $\Gamma$ (direct kinematics); that is, $P=$ $\Gamma(q)$ where $\Gamma: \mathbb{R}^{2} \longrightarrow \mathbb{R}^{3}$.

The orthosis structure proposed in this study can be modeled (considering the joint configuration) by introducing a second order nonlinear mathematical model corresponding to [49]

$$
\begin{gathered}
M(q(t)) \frac{d^{2} q(t)}{d t^{2}}+G(q(t))+C\left(q(t), \frac{d q(t)}{d t}\right) \frac{d q(t)}{d t} \\
+\psi\left(q(t), \frac{d q(t)}{d t}, t\right)=\tau(t)+\tau_{r}(t)
\end{gathered}
$$

where $q=\left[\begin{array}{ll}q_{1} & q_{2}\end{array}\right]^{\top}$. The matrix $M: \mathbb{R}^{2} \longrightarrow \mathbb{R}^{2 \times 2}$ stands for the inertia term, $G: \mathbb{R}^{2} \longrightarrow \mathbb{R}^{2}$ describes the gravitational effect terms, and $C(q, \dot{q}): \mathbb{R}^{2} \times \mathbb{R}^{2} \longrightarrow \mathbb{R}^{2 \times 2}$ is the so-called Coriolis matrix. The function $G$ must be Lipschitz while the Coriolis matrix must have a bounded consistent matrix. The uncertain term $\psi$ must be continuous with respect to its two first arguments. By the properties of the inertia matrix, the following assumption holds:

$$
0<m_{-} \leq \inf _{t \geq 0}\left\|M^{-1}(q)\right\|_{F}
$$

with $m_{-}$being a positive and constant scalar and $\|\cdot\|_{F}$ a consistent matrix norm.

The function $\psi: \mathbb{R}^{2} \times \mathbb{R}^{2} \times \mathbb{R}^{+} \longrightarrow \mathbb{R}^{2}$ contains all the perturbations and uncertainties affecting the model of the neck orthosis. Such term may include dried friction, nonmodeled internal interconnections between the rotor shaft in the actuator and the corresponding robot joint. The term $\tau=\left[\tau_{1}, \tau_{2}\right]^{\top}$ represents the input torque vector for both actuated articulations.

The term $\tau_{r}$ represents the effect of the constraint force induced by the patient that can oppose the orthosis movement. This opposition may come from the own pathology characteristics or the therapy evolution. Usually, this force satisfies the following model: $\tau_{r}(t)=J(q(t))^{\top} f_{\text {ext }}$ where $J(q(t))$ is the Jacobian that relates the terminal velocity of all arms in the orthosis to the time derivative of generalized coordinates $q$. Notice that $f_{\text {ext }}$ is the resistance function of the patient against the application of the induced movement by the orthosis.

The orthosis configuration, consisting of a two-degree-offreedom manipulator, justifies the Jacobian $J$ being bounded 


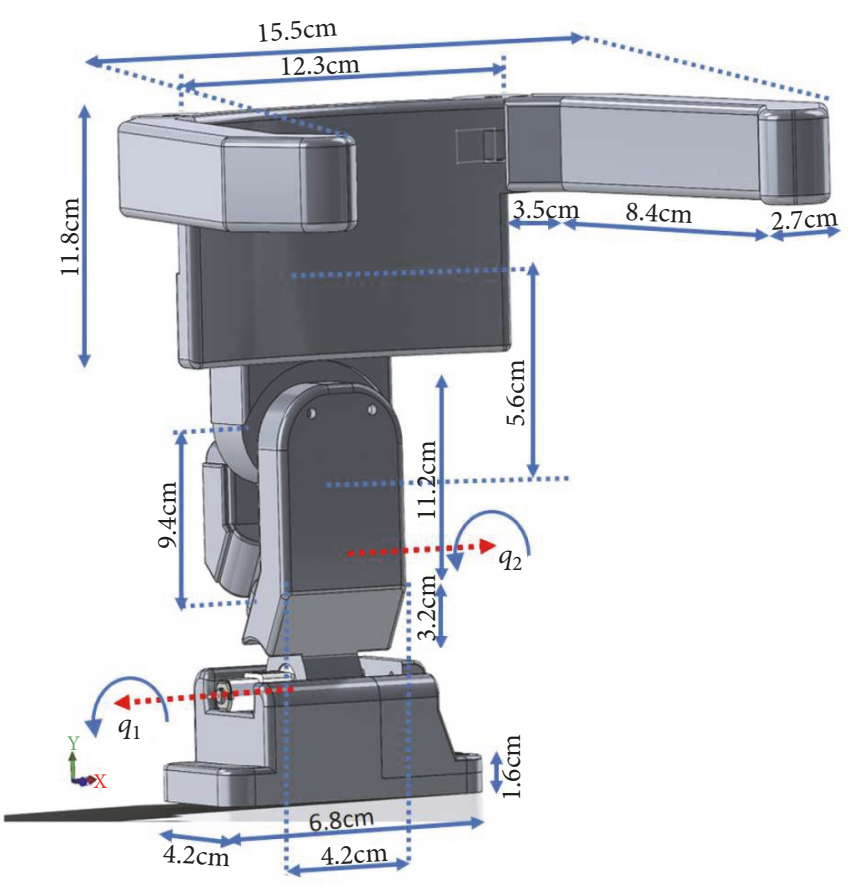

(a)

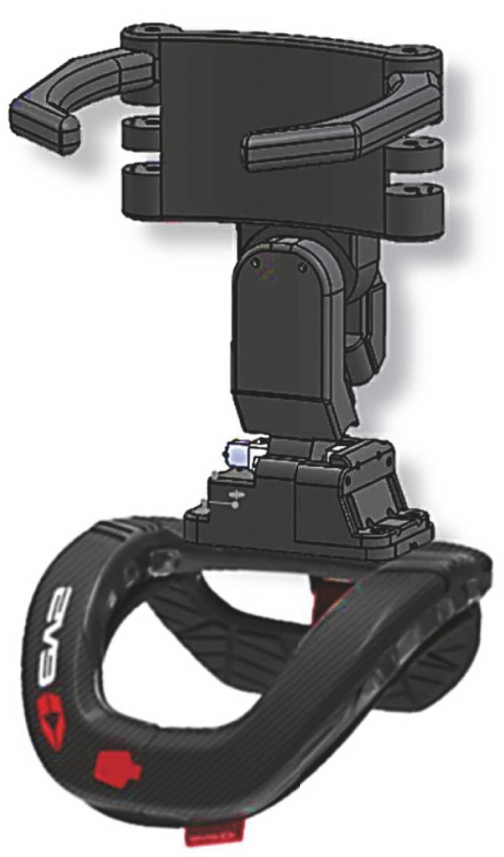

(b)

FIGURE 1: Computer assisted design model of the cervical spinal cord orthosis. (a) Dimensions of the elements included in the cervical orthosis as well as the generalized coordinates of movement. (b) General view of the cervical orthosis fixation over the supportive collar.

according to the result presented in [50]. In consequence, it is reasonable to introduce the following bound:

$$
\sup _{t \geq 0}\left\|J(q(t)) J^{\top}(q(t))\right\|_{F} \leq J^{+}
$$

with $J^{+}$being a positive scalar.

Notice that introducing the position coordinates and the operation space states enforces the necessity of defining the patient resistance, that is, the external force, which could be modeled as $f_{\text {ext }}=K_{s t}\left(q_{s}-q\right)$ where $q_{s} \in \mathbb{R}^{3}$ represents the position where the distal elements of the orthosis should move back if the external force disappears. The matrix $K_{s t} \in$ $\mathbb{R}^{2 \times 3}$ defines the patient stiffness, and it is usually unknown. Notice that the class of admissible external forces satisfies

$$
\sup _{t \geq 0}\left\|f_{\text {ext }}\right\| \leq f_{\text {ext }}^{+}
$$

where $f_{\text {ext }}^{+}$is a positive scalar.

The definition of the impedance adaptive control requires an additional part. When an external force acts on the set of end effectors (like the resistance enforced by the patient), the objective of the impedance controller is to cause the end effector to respond according to some defined dynamics. This part of the controller allows changing the dynamic response of the orthosis with respect to the patient.

For example, if the patient should not be forced by the orthosis, the desired dynamics can only track the patients movements and then reduce the impedance response to zero in the best case. On the contrary, if the orthosis must force the patient to complete a specific therapy, the desired dynamics can be adjusted to keep the resistance in an acceptable range. The bounds of this range should be proposed by a medical doctor.

\section{Adaptive Control Design for the Neck Orthosis Device}

The design of the automatic controller requires the definition of the model aimed at characterizing the human-machine interaction between the patient and the orthosis. This study considers two modes of human-machine interaction:

(i) Robot-in-charge: The robot plays the leading role to track the desired trajectory corresponding to the proposed therapy. At this condition, the human is not resistant to following the induced moment of the robotic orthosis. In this case, the interaction force $f_{\text {ext }}$ is not large.

(ii) Human-in-charge: The human exerts an increasing interaction force $f_{\text {ext }}$ on the robotic orthosis to take control actions. The robot becomes passive to complement human's intention and movement.

Notice that both orthosis-human interaction models are defining a class of interactions between the patient and the rehabilitation device, but fixing different purposes for the controller. The switch between modes of operation is automatically defined by estimating the interaction force between the orthosis and the patient.

If the robot-in-charge mode is active, the working environment of the orthosis operation is enforcing the patient's 


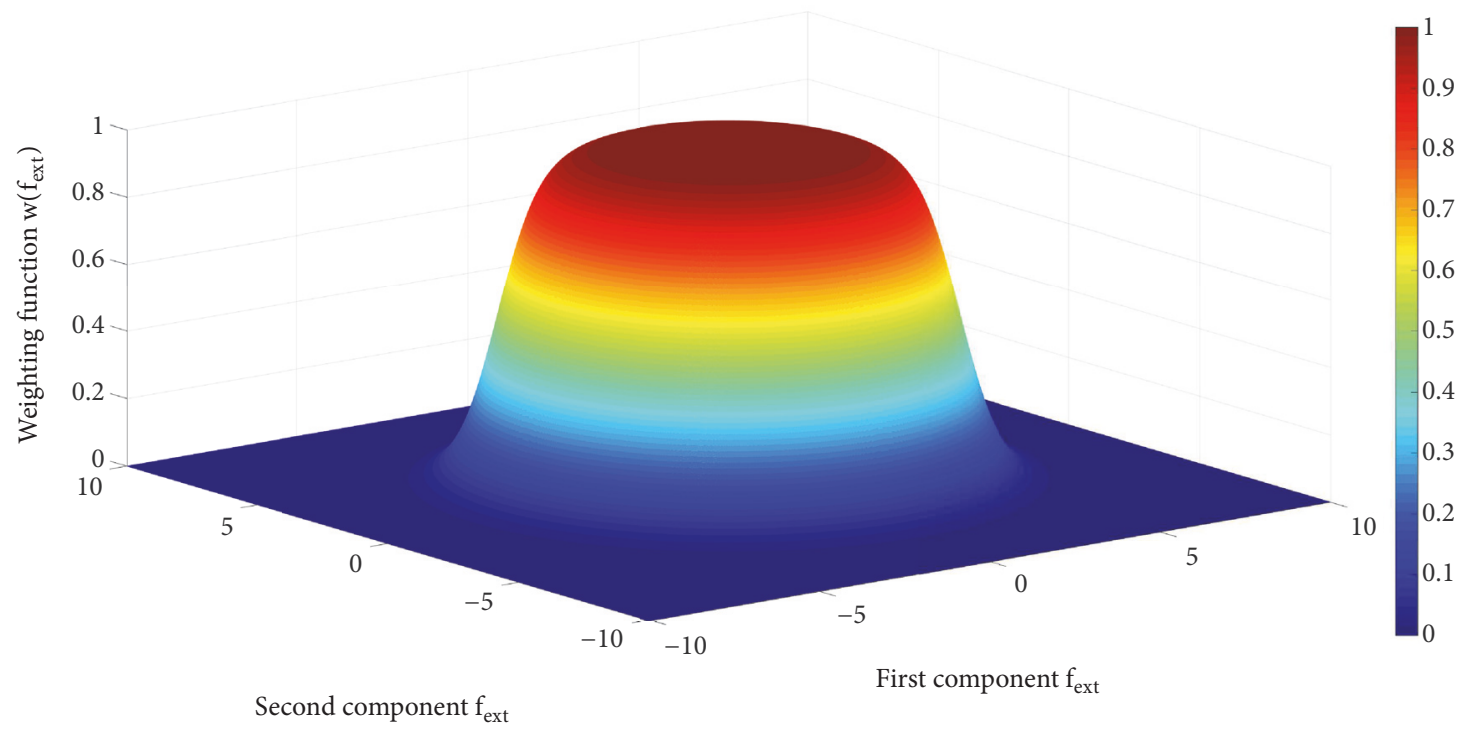

FIGURE 2: Weighting function $w\left(f_{\text {ext }}\right)$ calculated with $R=2.2, b=3.0$, and $c=2.0$.

neck movement without resistance. In this case, the robotic device can help the patient to track the desired trajectory accurately, without supervision from humans. In the second mode (human-in-charge), an external force increases by the patient's resistance to the robotic orthosis regular movement. Then, if the force $f_{\text {ext }}$ grows over a predefined value, then it is expected that the device stops its movement to avoid forcing the cervical vertebra beyond a safe condition proposed by the treating physician.

The dual human-robot (patient-orthosis) interaction modes can also be applied in sensory-feedback robotic orthosis to improve the treatment performance, without inducing unsafe movement of the cervical region. The orthosis functions within the robot-in-charge mode when it performs the vertebra manipulation if the external force sensory-feedback reports a small patient's resistance. However, if the patient takes control in the human-in-charge mode because the patient offers resistance to the execution of the therapy, the sensory-feedback reports an external force moving outside a predefined limiting zone.

The dual interaction depends on the measurement of the external force with certain degree of accuracy. In general, the measurement of such force can be obtained directly from a sensor placed at some adequate position in the orthosis structure. An alternative option is using an indirect measurement of the external force by introducing a current sensor on the actuator circuit. There are several studies estimating the resistance force of the robot movement by implementing a class of observers based on the current measurements. In this case, there is the assumption that $f_{\text {ext }}$ can be measured online by the corresponding force sensor.

The estimation of $f_{\text {ext }}$, named $\widehat{f}_{e}$, is obtained by direct measurement of the consumed current at each actuator $I_{i}, i=$ 1,2 , and then the nonlinear transformation $\Omega$ defines $\widehat{f}_{e}=$
$\Omega\left(I_{1}, I_{2}\right)$. The variation of this force $\widehat{f}_{e}$ defines the transition between both modes of human-machine interaction.

According to [51], it is necessary to introduce a monitoring function describing the transition between each interaction mode, namely, $h\left(\widehat{f}_{e}\right)$, which is proposed as follows:

$$
h\left(\widehat{f}_{e}\right)=\left\|\widehat{f}_{e}\right\|^{2}-R^{2}
$$

with $R$ being a positive constant defined by the designer. Notice that it may be helpful to define this function; if the orthosis could track the reference trajectory without patient's resistance, then $h\left(\widehat{f}_{e}\right)<0$. On the other hand, if the patient starts increasing its resistance against the orthosis performance, then $\left(\widehat{f}_{e}\right)$ increases from a negative to a positive value.

Considering the monitoring function $\left(\widehat{f}_{e}\right)$, a weighting function can be suggested to characterize the mode transition numerically. This study used the following vector-valued weighting function.

$$
w\left(\widehat{f}_{e}\right)=1-\frac{1}{1+b e^{-c \cdot h\left(\widehat{f}_{e}\right)}}
$$

In (6), the parameters $b$ and $c$ are positive constant scalars which can be adjusted considering the expected transition between interaction modes for each patient. Figure 2 shows the evaluation of function $w$ with respect to the components of $\widehat{f}_{e}$. The smoothness of function (6) is modifiable by adjusting the values of parameters $b$ and $c$.

The function $w$ depends on the function $\hat{f}_{e}$ which can be estimated by the current measurements on the actuators. The corresponding relationships can be obtained from adequate characterizations which are proposed in the experimental section following the general ideas proposed in [52-54]. 
Based on the weighting function, it is possible to define the reference trajectories considering the desired position of the reference point $P_{d}$. The time-dependent sequence of $P_{d}$ defines the movements of the central section for the robotic orthosis. In consequence, the dynamics of the desired angular displacement $q_{i, d}$ can be estimated as

$$
\begin{aligned}
\frac{d}{d t} q_{d}(t)= & w\left(\widehat{f}_{e}(t)\right) \Gamma^{+}(q(t)) \frac{d}{d t} P_{d}(t) \\
& -w\left(\widehat{f}_{e}(t)\right) \Gamma^{+}(q(t)) \Delta P(t)
\end{aligned}
$$

where $\Gamma^{+}(q(t))$ is the pseudoinverse of $\Gamma(q(t))$ and $\Delta P=$ $P-P_{d}$. Indeed, the desired angular positions come from the inverse transformation related to the kinematics relation between the position of the end-effect position and the angular variations. Notice that the product of $\Gamma^{+} P_{d}$ is $q_{d}$. Then, the only equilibrium point is 0 for $\Delta P$ which is indeed the goal of the controller.

3.1. Tracking Error Dynamics. Introduce the tracking error dynamics $\delta$ defined as $\delta=q-q_{d}$. The application of the state variable theory suggests introducing the states $\delta_{a}=\delta$ and $\delta_{b}=(d / d t) \delta$, and then the following identities hold.

$$
\begin{aligned}
& \frac{d}{d t} \delta_{a}(t)=\delta_{b}(t) \\
& \frac{d}{d t} \delta_{b}(t)=F\left(q(t), \frac{d}{d t} q(t)\right)+G(q(t)) \tau(t) \\
& +\Phi\left(q(t), \frac{d}{d t} q(t), t\right)+M^{-1}(q(t)) J(q(t))^{\top} \\
& \cdot f_{e x t}(t)-\frac{d}{d t}\left[w\left(\widehat{f}_{e}(t)\right) \Gamma^{+}(q(t)) \frac{d}{d t} P_{d}(t)\right. \\
& \left.-w\left(\widehat{f}_{e}(t)\right) \Gamma^{+}(q(t)) \Delta P(t)\right]
\end{aligned}
$$

In (8), the function $F: \mathbb{R}^{2} \times \mathbb{R}^{2} \longrightarrow \mathbb{R}^{2}$ corresponds to $F(q,(d / d t) q)=-M^{-1}(q)(G(q)+C(q,(d / d t) q)(d / d t) q)$, the function $G: \mathbb{R}^{2} \longrightarrow \mathbb{R}^{2 \times 2}$ corresponds to $G(q)=-M^{-1}(q)$, and the function $\Phi: \mathbb{R}^{2} \times \mathbb{R}^{2} \times \mathbb{R}^{2} \longrightarrow \mathbb{R}^{2}$ satisfies the following identity $\Phi(q,(d / d t) q, t)=-M^{-1}(q) \psi(q(t),(d / d t) q(t), t)$.

The function $F$ satisfies the following inequality:

$$
\left\|F\left(q, \frac{d}{d t} q\right)\right\| \leq f_{0}+f_{1}\left\|q_{v}\right\|
$$

where $f_{0}$ and $f_{1}$ are positive constant scalars and $q_{v}=$ $\left[q^{\top},(d / d t) q^{\top}\right]^{\top}$.

In equivalent form, the uncertainties term $\Phi$ satisfies

$$
\left\|\Phi\left(q, \frac{d}{d t} q, t\right)\right\| \leq \phi_{0}+\phi_{1}\left\|q_{v}\right\|
$$

where $\phi_{0}$ and $\phi_{1}$ are positive constant scalars.

The suggested design of the reference trajectories $q_{d}$ aggregates both modes of operation. The following analysis demonstrates how each mode operates over the orthosispatient interaction. (a) When the orthosis is completely ruling the movement (no patient resistance), that is, $w\left(\hat{f}_{e}(t)\right)=1$, the dynamics of the orthosis-patient dynamics satisfies (with $f_{\text {ext }}=0$ ) the following.

$$
\begin{aligned}
& \frac{d}{d t} \delta_{a}(t)=\delta_{b}(t) \\
& \frac{d}{d t} \delta_{b}(t) \\
& =F\left(q(t), \frac{d}{d t} q(t)\right)+G(q(t)) \tau(t) \\
& \quad+\Phi\left(q(t), \frac{d}{d t} q(t), t\right) \\
& \quad-\frac{d}{d t}\left[\Gamma^{+}(q(t)) \frac{d}{d t} P_{d}(t)-\Gamma^{+}(q(t)) \Delta P(t)\right]
\end{aligned}
$$

The control problem formulated for this case implies the design of $\tau=\tau_{t r}$ in such a way that $\delta=\left[\begin{array}{ll}\delta_{a} & \delta_{b}\end{array}\right]^{\top}$ must be zero; that is, the articulation angles track the desired trajectory.

(b) If the patient is resistant to the orthosis action, then $w\left(\hat{f}_{e}(t)\right)=0$, making sure also that $\tau$ must only compensate the uncertain term $\Phi$, namely, $\tau=\tau_{c}$. Then, the dynamics of the orthosis-patient dynamics satisfies (with $f_{\text {ext }} \neq 0$ ) the following.

$$
\begin{aligned}
\frac{d}{d t} \delta_{a}(t)= & \delta_{b}(t) \\
\frac{d}{d t} \delta_{b}(t)= & F\left(q(t), \frac{d}{d t} q(t)\right)+G(q(t)) \tau_{c}(t) \\
& +\Phi\left(q(t), \frac{d}{d t} q(t), t\right) \\
& +M^{-1}(q(t)) J(q(t))^{\top} f_{\text {ext }}(t)
\end{aligned}
$$

The control problem in this second case when the patient is ruling the orthosis action $\left(w\left(\hat{f}_{e}(t)\right)=1\right)$ must provide the fact that $\tau_{c}(t)=\Phi(q(t),(d / d t) q(t), t), \forall t \geq T_{c}$. Notice that if the compensation is exact, then the orthosis-patient interaction satisfies the following dynamics.

$$
\begin{aligned}
\frac{d}{d t} \delta_{a}(t)= & \delta_{b}(t) \\
\frac{d}{d t} \delta_{b}(t)= & F\left(q(t), \frac{d}{d t} q(t)\right) \\
& +M^{-1}(q(t)) J(q(t))^{\top} f_{\text {ext }}(t)
\end{aligned}
$$

The dynamic model presented in (13) is a damping system in the sense that the patient is taking the control of the orthosis by using $J(q(t))^{\top} f_{\text {ext }}(t)$ as the external torque.

The patient's impedance against the robotic orthosis may vary from a comparatively large to a small value. The impedance variation forces the transition from one model 
to the other one (from the orthosis-in-charge to the patientin-charge mode). Therefore, there is a smooth combination of the situation when the human can guide the orthosis movement and the case when the robotic device is playing the assistance forces. The transition scenario $0<w\left(\widehat{f}_{e}(t)\right)<$ 1 defines the so-called shared-control scenarios combining the patient control actions and the orthosis robot controlled realization. The proportion of transition stage along this transition stage can be adjusted by setting the parameters in the weighting function. The variation of the radius $R$ defines specific cases for different patients. If the radius is set large enough, then the orthosis plays a more active role along the transition stage, but if the patient should be allowed to take the device control, the $R$ must be decreased. This modification depends on the rehabilitation stage for each patient. In the case of fully paralysed vertebral movement, the orthosis must take the leading role to control the stability of the neck's spinal cord section. Otherwise, if the patient is recovering the movement abilities, then the $R$ value can be decreased.

\subsection{Design of the Reference Trajectories for the Patient's}

Therapy. The definition of the reference trajectory for the central point in the orthosis structure, that is, $P_{d}$, requires a particular technique. Usually, this part of the process receives the name of planning trajectory, which can be solved by implementing the so-called Bezier polynomials. In this study, this planning process considered a suitable interpolation method based on nonlinear differentiable functions. In this case, this part of the process also uses sigmoid functions.

The efficient design of the reference trajectories implies the explicit solution of suitable algebraic systems of equations which defines remarkable sections of the planning process (defined by the time instants when the reference trajectory is inflected). Such solution is time consuming with high computational complexity.

The simpler composition of sigmoid functions may overcome these two drawbacks. Such composition uses the initial and final values of the sigmoid functions as well as the so-called transition time for each inflection section $t_{k}$. The sigmoid function considered in this part of the study obeys

$$
s(t)=\frac{p_{1}}{1+p_{2} e^{\left(t-t_{k}\right)}}+p_{3}, \quad \forall t \in\left[t_{k}, t_{k+1}\right]
$$

where the parameters $p_{1}, p_{2}$, and $p_{3}$ are positive constant scalars. The procedure aimed at constructing a reference trajectory when a particular element must move from an initial position $\zeta_{0}$ to a final position $\zeta_{f}$ in a time period of $d_{t}=t_{k+1}-t_{k}$ seconds, can be explained as follows.

(i) Fix the value $p_{1} /\left(1+p_{2}\right)+c=\zeta_{0}$.

(ii) Fix the value $p_{1} /\left(1+p_{2} e^{d_{t}}\right)+p_{3}=\zeta_{f}$.

(iii) Fix the value $p_{1} /\left(1+p_{2} e^{0.5 d_{t}}\right)+p_{3}=0.5\left(\zeta_{f}-\zeta_{0}\right)$.

The solution of this algebraic system yields the thirdorder polynomial for the parameter $p_{2}$ :

$$
p_{2}^{3}+w_{2} p_{2}^{2}+w_{1} p_{2}+w_{0}=0
$$

where $w_{2}, w_{1}$, and $w_{0}$ are straightforwardly estimated. According to [55], if $\rho_{1}^{2}-4 \rho_{0}^{3} \geq 0\left(\rho_{0}=w_{2}^{2}-3 w_{1}, \rho_{1}=\right.$ $\left.2 w_{2}^{3}-9 w_{1} w_{2}+27 w_{0}\right)$, then $p_{2}$ can be explicitly calculated as

$$
\begin{aligned}
& p_{2}=-\frac{w_{2}+C_{1}+C_{2}}{3} \\
& C_{1}=\left(\frac{\rho_{1}-\sqrt{\rho_{1}^{2}-4 \rho_{0}^{3}}}{2}\right)^{1 / 3} ; C_{2}=\left(\frac{\rho_{1}+\sqrt{\rho_{1}^{2}-4 \rho_{0}^{3}}}{2}\right)^{1 / 3}
\end{aligned}
$$

which is the real root of cubic equation (15). Based on the result for $p_{2}$, the value of $p_{1}$ is as follows.

$$
p_{1}=\frac{0.5\left(\zeta_{f}-\zeta_{0}\right)(1+b)\left(1+b e^{d_{t}}\right)}{b\left(1-e^{d_{t}}\right)}
$$

The parameter $p_{3}$ is as follows.

$$
p_{3}=\zeta_{0}-\frac{a}{1+b}
$$

The time derivative of the reference trajectory can be directly calculated from the sigmoid function. In consequence, $P_{d}$ and $(d / d t) P_{d}$ can be used in the controller design.

3.3. The Adaptive Controller. The controller structure to solve the orthosis-patient interaction process satisfies the following mixed structure.

$$
\begin{aligned}
& \tau(t)=G^{-1}(q(t)) \\
& \cdot H^{-1}\left(w\left(\widehat{f}_{e}(t)\right) \tau_{t r}(t)+\left[1-w\left(\hat{f}_{e}(t)\right)\right] \tau_{c}(t)\right)
\end{aligned}
$$

To complete the control design, let us define the sliding vector $S$ as

$$
S(t)=\delta_{a}(t)+H \delta_{b}(t)
$$

with $H \in \mathbb{R}^{2 \times 2}$ being a positive definite matrix.

The dynamics of $S$ satisfies the following.

$$
\frac{d}{d t} S(t)=\delta_{b}(t)+H \frac{d}{d t} \delta_{b}(t)
$$

Here $(d / d t) \delta_{b}(t)$ comes from model (8). Notice that the dynamics of $S$ depends on the mode executed by the orthosispatient interaction.

The proposed controller uses the following adaptive sliding mode theory:

$$
\begin{gathered}
\tau_{t r}(t)=-K_{t r}(t)\left[1+\lambda \sqrt{\epsilon+\left\|q_{v}\right\|^{2}}\right] \frac{S(t)}{\|S(t)\|}-\Pi_{t r} \\
\tau_{c}(t)=-K_{c}(t)\left[1+\lambda \sqrt{\epsilon+\left\|q_{v}\right\|^{2}}\right] \frac{S(t)}{\|S(t)\|}-\Pi_{c} \\
\Pi_{t r}=\delta_{b}(t)-H \frac{d}{d t}\left[\Gamma^{+}(q(t)) \frac{d}{d t} P_{d}(t)-\Gamma^{+}(q(t)) \Delta P(t)\right] \\
\Pi_{c}=\delta_{b}(t)+H M^{-1}(q(t)) J(q(t))^{\top} f_{\text {ext }}(t)
\end{gathered}
$$




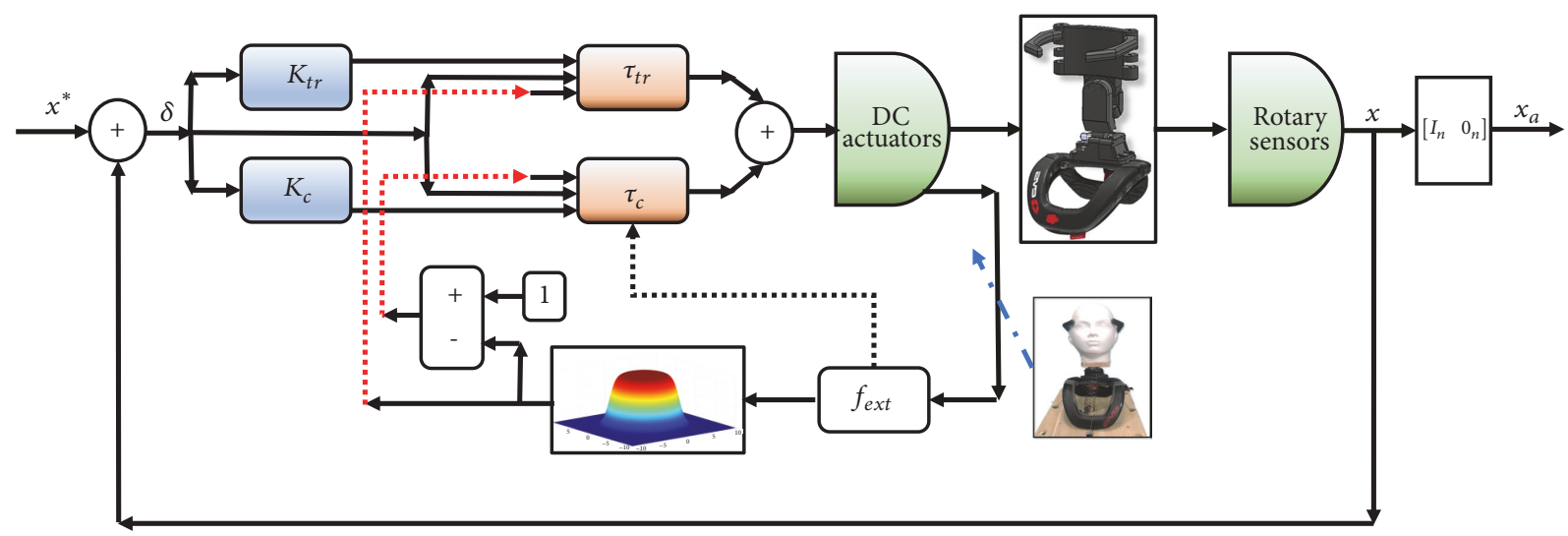

FIGURE 3: Simplified diagram of the proposed adaptive controller.

where $\lambda \geq 0, \epsilon>0$, and $K_{t r} \in \mathbb{R}^{2 \times 2}$ and $K_{c} \in \mathbb{R}^{2 \times 2}$ are the adaptive controller matrix gains satisfying:

$$
\begin{aligned}
\frac{d}{d t} K_{t r}(t)= & \left(\gamma_{0}+\gamma_{1}\left\|q_{v}\right\|\right) K_{t r}(t) \operatorname{sign}_{e}(S(t)) \\
& -M\left[K_{t r}(t)-K_{t r}^{+}\right]_{+} \\
& +M\left[\mu_{t r}-K_{t r}(t)\right]_{+} \\
\frac{d}{d t} K_{c}(t)= & \left(\gamma_{0}+\gamma_{1}\left\|q_{v}\right\|\right) K_{c}(t) \operatorname{sign}_{e}(S(t)) \\
& -M\left[K_{c}(t)-K_{c}^{+}\right]_{+} \\
& +M\left[\mu_{c}-K_{c}(t)\right]_{+}
\end{aligned}
$$

with $\operatorname{sign}_{e}(S(t)):=|S(t) /\|S(t)\||-\alpha$, where $\operatorname{sign}_{e}(S(t))$ is the vector formed with the components of the filtered signal associated with $S_{i} /\|S(t)\|, i=1,2$. Figure 3 shows a simplified diagram of the proposed control including the estimation of the external force $f_{\text {ext }}$ and the simultaneous calculus of the time-varying gains. The state dependence of the gains (23) motivates labeling the controller (22) as an adaptive structure. Notice that this description tries to highlight the difference between the proposed controller and the classical ones where a fixed value of gain may counteract the perturbations/uncertainties effect, without taking care of the operation mode.

This study considers the application of a direct estimation of the external force $f_{\text {ext }}$ depending on the currents measured at the DC actuators. Therefore, this study did not consider a direct estimation of a second order model for the $f_{\text {ext }}$. Clearly this is a potential future trend on our orthosis device, but this study considered a simpler form which can be solved with the additional instrumentation of the current sensors as well as the previous characterization of the relationship between external forces and actuators currents.

The motivation of using adaptive form of sliding mode controller is the necessity of having an accurate estimation of the uncertainties affecting the orthosis-patient system. Even more, some recent works are showing that some adaptive forms of sliding controllers offer robustness against some kind of perturbations (bounded with restricted derivative) as well as the finite-time convergence. Originally, the introduction of adaptive gains motivated the application of discontinuous controllers (sliding mode type) with the smaller attainable chattering effect. The application of the equivalent control concept also offers the advantage of recovering an accurate estimation of the uncertainties affecting the controlled system. Such estimation can be further used as part of the control as a compensation term.

This section presents the main result of this study in the following lemma.

Lemma 1. Consider the closed-loop dynamics of the active neck orthosis under the assumption of a human-robot interaction given in (8) satisfying restrictions (9) and (10) with the adaptive controller (19) adjusted with the time-dependent gains $K_{t r}$ and $K_{c}$ given in (23). If the tuning parameters $\mu_{t r}$ and $\lambda$ of the gains $K_{t r}$ and $K_{c}$ given in (23) satisfy

$$
\begin{aligned}
\mu_{t r} & =\|H\|_{F} \eta_{0}+\rho_{t r} \\
\lambda & =\frac{\eta_{1}}{\mu_{t r}} \\
\mu_{c} & =\mu_{t r}
\end{aligned}
$$

with $\eta_{0}=f_{0}+\phi_{0}, \eta_{1}=f_{1}+\phi_{1}$, and $\rho_{t r}>0$, then the origin is a finite-time stable equilibrium point for the tracking error $\delta$.

Proof. The proof of this lemma is presented in three sections: the first considers the case when the robotic orthosis is handling the neck without patient resistance, the second considers the case when the patient is taking control of the human-robot interaction, and the third analyzes the transition phase between the two previous cases

Orthosis Control Mode. Based on the definition of the proposed controller, the time derivative of the sliding vector satisfies the following.

$$
\begin{aligned}
& \frac{d}{d t} S(t)=\delta_{b}(t)+H F\left(q(t), \frac{d}{d t} q(t)\right) \\
& \quad+\left(w\left(\widehat{f}_{e}(t)\right) \tau_{t r}(t)+\left[1-w\left(\widehat{f}_{e}(t)\right)\right] \tau_{c}(t)\right)
\end{aligned}
$$




$$
\begin{aligned}
& +H \Phi\left(q(t), \frac{d}{d t} q(t), t\right) \\
& +H M^{-1}(q(t)) J(q(t))^{\top} f_{\text {ext }}(t) \\
& -H \frac{d}{d t}\left[w\left(\widehat{f}_{e}(t)\right) \Gamma^{+}(q(t)) \frac{d}{d t} P_{d}(t)\right. \\
& \left.-w\left(\widehat{f}_{e}(t)\right) \Gamma^{+}(q(t)) \Delta P(t)\right]
\end{aligned}
$$

Let us consider the case when the orthosis is ruling the neck's movement; then $w\left(\widehat{f}_{e}(t)\right)=1$, and then (25) yields the following.

$$
\begin{aligned}
& \frac{d}{d t} S(t) \\
& =\delta_{b}(t)+H F\left(q(t), \frac{d}{d t} q(t)\right)+\tau_{t r}(t) \\
& \quad+H \Phi\left(q(t), \frac{d}{d t} q(t), t\right) \\
& \quad-H \frac{d}{d t}\left[\Gamma^{+}(q(t)) \frac{d}{d t} P_{d}(t)-\Gamma^{+}(q(t)) \Delta P(t)\right]
\end{aligned}
$$

The substitution of $\tau_{t r}$ leads to the following.

$$
\begin{aligned}
\frac{d}{d t} S(t)= & H F\left(q(t), \frac{d}{d t} q(t)\right) \\
& +H \Phi\left(q(t), \frac{d}{d t} q(t), t\right) \\
& -K_{t r}(t)\left[1+\lambda \sqrt{\epsilon+\left\|q_{v}\right\|^{2}}\right] \frac{S(t)}{\|S(t)\|}
\end{aligned}
$$
tion.

Now let us propose the energetic (Lyapunov-like) func-

$$
V_{t r}(S)=0.5\|S\|^{2}
$$

The function $V_{t r}$ is positive definite, $V_{t r}(0)=0$, and radially unbounded. The Lie-derivative of $V_{1}$ over the trajectories of (27) is as follows.

$$
\begin{aligned}
& \frac{d}{d t} V_{t r}(S(t))=S^{\top}(t)\left[H F\left(q(t), \frac{d}{d t} q(t)\right)\right. \\
& \quad+H \Phi\left(q(t), \frac{d}{d t} q(t), t\right) \\
& \left.\quad-K_{t r}(t)\left[1+\lambda \sqrt{\epsilon+\left\|q_{v}\right\|^{2}}\right] \frac{S(t)}{\|S(t)\|}\right]
\end{aligned}
$$

Using inequalities (10) and (9), one gets the following.

$$
\begin{aligned}
\frac{d}{d t} V_{t r}(S(t)) \leq & \|H\|_{F}\left(\eta_{0}+\eta_{1}\left\|q_{v}\right\|\right)\|S(t)\| \\
& -K_{t r}(t)\left[1+\lambda \sqrt{\epsilon+\left\|q_{v}\right\|^{2}}\right]\|S(t)\|
\end{aligned}
$$

Notice the following.

$$
\begin{gathered}
\|H\|_{F}\left(\eta_{0}+\eta_{1}\left\|q_{v}\right\|\right)-K_{t r}(t)\left[1+\lambda \sqrt{\epsilon+\left\|q_{v}\right\|^{2}}\right] \\
<\|H\|_{F}\left(\eta_{0}+\eta_{1}\left\|q_{v}\right\|\right)-\mu_{t r}\left[1+\lambda\left\|q_{v}\right\|\right]
\end{gathered}
$$

In view of the parameter selection in (24), then $(d / d t) V_{t r}(S(t)) \leq 0$, and then

$$
\frac{d}{d t} V_{t r}(S(t)) \leq-\sqrt{2} \rho_{t r} \sqrt{V_{t r}(S(t))}
$$

The standard procedure can be used to prove that $V_{t r}(S(t))=0, \forall t \geq T_{t r}=\rho_{t r}^{-1} \sqrt{V_{t r}(S(0))}$.

Patient Control Mode. Let us consider the case when $w\left(\widehat{f}_{e}(t)\right)=0$; then $(25)$ yields the following.

$$
\begin{aligned}
\frac{d}{d t} S(t)= & \delta_{b}(t)+H F\left(q(t), \frac{d}{d t} q(t)\right)+\tau_{c}(t) \\
& +H \Phi\left(q(t), \frac{d}{d t} q(t), t\right) \\
& +H M^{-1}(q(t)) J(q(t))^{\top} f_{\text {ext }}(t)
\end{aligned}
$$

The application of the compensating controller $\tau_{c}$ yields the following.

$$
\begin{aligned}
\frac{d}{d t} S(t)= & H F\left(q(t), \frac{d}{d t} q(t)\right) \\
& +H \Phi\left(q(t), \frac{d}{d t} q(t), t\right) \\
& -K_{c}(t)\left[1+\lambda \sqrt{\epsilon+\left\|q_{v}\right\|^{2}}\right] \frac{S(t)}{\|S(t)\|}
\end{aligned}
$$

Considering the value of $\mu_{c}$ and following a similar procedure to the one shown in the case when $w\left(\widehat{f}_{e}(t)\right)=1$, then $(d / d t) V_{c}(S(t)) \leq 0$ with $V_{c}(S)=0.5\|S\|^{2}$, and then

$$
\frac{d}{d t} V_{c}(S(t)) \leq-\sqrt{2} \rho_{c} \sqrt{V_{c}(S(t))}
$$

The standard procedure can be used to prove that $V_{c}(S(t))=0, \forall t \geq T_{c}=\rho_{c}^{-1} \sqrt{V_{c}(S(0))}$.

Transition Phase. If the transition process is considered and both controllers $\tau_{t r}$ and $\tau_{c}$ are applied, then (25) yields the following. 


$$
\begin{aligned}
& \frac{d}{d t} S(t)=\delta_{b}(t)+H F\left(q(t), \frac{d}{d t} q(t)\right)+w\left(\widehat{f}_{e}(t)\right) \\
& \cdot\left(-K_{t r}(t)\left[1+\lambda \sqrt{\epsilon+\left\|q_{v}\right\|^{2}}\right] \frac{S(t)}{\|S(t)\|}-\delta_{b}(t)\right. \\
& \left.+H \frac{d}{d t}\left[\Gamma^{+}(q(t)) \frac{d}{d t} P_{d}(t)-\Gamma^{+}(q(t)) \Delta P(t)\right]\right) \\
& +\left[1-w\left(\widehat{f}_{e}(t)\right)\right] \\
& \cdot\left(-K_{c}(t)\left[1+\lambda \sqrt{\epsilon+\left\|q_{v}\right\|^{2}}\right] \frac{S(t)}{\|S(t)\|}-\delta_{b}(t)\right. \\
& \left.-H M^{-1}(q(t)) J(q(t))^{\top} f_{\text {ext }}(t)\right) \\
& +H \Phi\left(q(t), \frac{d}{d t} q(t), t\right)+H M^{-1}(q(t)) J(q(t))^{\top} \\
& \cdot f_{\text {ext }}(t)-H \frac{d}{d t}\left[w\left(\widehat{f}_{e}(t)\right) \Gamma^{+}(q(t)) \frac{d}{d t} P_{d}(t)\right. \\
& \left.-w\left(\widehat{f}_{e}(t)\right) \Gamma^{+}(q(t)) \Delta P(t)\right]
\end{aligned}
$$

The reorganization of (36) justifies the following differential equation.

$$
\begin{aligned}
\frac{d}{d t} S(t) & =H F\left(q(t), \frac{d}{d t} q(t)\right) \\
+ & H \Phi\left(q(t), \frac{d}{d t} q(t), t\right)+-\left(w\left(\widehat{f}_{e}(t)\right) K_{t r}(t)\right. \\
+ & {\left.\left[1-w\left(\widehat{f}_{e}(t)\right)\right] K_{c}(t)\right)\left[1+\lambda \sqrt{\epsilon+\left\|q_{v}\right\|^{2}}\right] } \\
& \cdot \frac{S(t)}{\|S(t)\|}+w\left(\widehat{f}_{e}(t)\right) \\
\cdot & H M^{-1}(q(t)) J(q(t))^{\top} f_{\text {ext }}(t)+w\left(\widehat{f}_{e}(t)\right) \\
\cdot & H \frac{d}{d t}\left[\Gamma^{+}(q(t)) \frac{d}{d t} P_{d}(t)-\Gamma^{+}(q(t)) \Delta P(t)\right] \\
- & H \frac{d}{d t}\left[w\left(\widehat{f}_{e}(t)\right) \Gamma^{+}(q(t)) \frac{d}{d t} P_{d}(t)\right. \\
- & \left.w\left(\widehat{f}_{e}(t)\right) \Gamma^{+}(q(t)) \Delta P(t)\right]
\end{aligned}
$$

Considering that the function $w\left(\widehat{f}_{e}(t)\right)$ is explicitly determined in time, then (38) is equivalent to the following.

$$
\begin{aligned}
& \frac{d}{d t} S(t)=H F\left(q(t), \frac{d}{d t} q(t)\right) \\
& \quad+H \Phi\left(q(t), \frac{d}{d t} q(t), t\right) \\
& \quad-\left(w\left(\hat{f}_{e}(t)\right) K_{t r}(t)+\left[1-w\left(\widehat{f}_{e}(t)\right)\right] K_{c}(t)\right)
\end{aligned}
$$

$$
\begin{aligned}
& \cdot\left[1+\lambda \sqrt{\epsilon+\left\|q_{v}\right\|^{2}}\right] \frac{S(t)}{\|S(t)\|}+w\left(\widehat{f}_{e}(t)\right) \\
& \cdot H M^{-1}(q(t)) J(q(t))^{\top} f_{\text {ext }}(t)
\end{aligned}
$$

Now let us propose the energetic (Lyapunov-like) function.

$$
V_{m t}(S)=0.5\|S\|^{2}
$$

This function is also positive definite, $V_{m t}(0)=0$, and radially unbounded. The Lie-derivative of $V_{m t}$ over the trajectories of (38) corresponds to the following.

$$
\begin{aligned}
& \frac{d}{d t} V_{m t}(S(t))=S^{\top}(t) \\
& \cdot\left[H F\left(q(t), \frac{d}{d t} q(t)\right)+H \Phi\left(q(t), \frac{d}{d t} q(t), t\right)\right] \\
& +S^{\top}(t) \\
& \cdot\left[w\left(\widehat{f}_{e}(t)\right) H M^{-1}(q(t)) J(q(t))^{\top} f_{\text {ext }}(t)\right] \\
& +\left(w\left(\widehat{f}_{e}(t)\right) K_{t r}(t)+\left[1-w\left(\widehat{f}_{e}(t)\right)\right] K_{c}(t)\right) \\
& \cdot\left[1+\lambda \sqrt{\epsilon+\left\|q_{v}\right\|^{2}}\right]\|S(t)\|
\end{aligned}
$$

Using inequalities (10) and (9) as well as the assumptions regarding the bounds for the Jacobian $J$ (3) and the $f_{\text {ext }}(4)$, one gets the following.

$$
\begin{aligned}
& \frac{d}{d t} V_{t r}(S(t)) \leq\|H\|_{F}\left(\eta_{0}+m^{-}\left(J^{+}\right)^{1 / 2} f_{\text {ext }}^{+}+\eta_{1}\left\|q_{v}\right\|\right) \\
& \cdot\|S(t)\| \\
& -\left(w\left(\widehat{f}_{e}(t)\right) K_{t r}(t)+\left[1-w\left(\widehat{f}_{e}(t)\right)\right] K_{c}(t)\right) \\
& \cdot\left[1+\lambda \sqrt{\epsilon+\left\|q_{v}\right\|^{2}}\right]\|S(t)\|
\end{aligned}
$$

Notice the following.

$$
\begin{gathered}
\|H\|_{F}\left(\eta_{0}+\eta_{1}\left\|q_{v}\right\|\right)-K_{t r}(t)\left[1+\lambda \sqrt{\epsilon+\left\|q_{v}\right\|^{2}}\right] \\
<\|H\|_{F}\left(\eta_{0}+\eta_{1}\left\|q_{v}\right\|\right)-\mu_{t r}\left[1+\lambda\left\|q_{v}\right\|\right]
\end{gathered}
$$

Then, in view of the values for $\mu_{t r}$ and $\lambda,(d / d t) V_{t r}(S(t)) \leq$ 0 , yielding the following.

$$
\frac{d}{d t} V_{t r}(S(t)) \leq-\sqrt{2} \rho_{t r} \sqrt{V_{t r}(S(t))}
$$

The standard procedure can be used to prove that $V_{t r}(S(t))=0, \forall t \geq T_{t r}=\rho_{t r}^{-1} \sqrt{V_{t r}(S(0))}$.

Considering that all the three phases (orthosis control, patient control, and transition) admits a Lyapunov function and all three functions served to prove that the origin is a robust finite-time stable equilibrium point, then, it is possible to argument that, for all possible variants of $w$, this stability characteristic holds. 
3.4. The Output Feedback Version of the Adaptive Controller. The adaptive controller proposed above can be implemented under a technical restriction, and the angular displacement and the angular velocity must be measured simultaneously, yielding the estimation of the tracking error and its derivative on-line; that is, $(d / d t) e(t)$ and $e(t)$ can be applied directly in the calculus of $S$.

However, these variables are rarely measured at the same time, unless important resources investment is realized. An alternative is using a simple and effective robust and exact observer or differentiator aimed at estimating a suitable as well as accurate approximation of the tracking error time derivative [56].

In opposition to other sliding modes algorithms (second order), the super twisting (ST) can be applied in the case of systems with relative degree equal to one with respect to the chosen output [57]. The ST algorithm application as a robust differentiator is described as follows. Consider that $w_{1}(t)=r(t)$ where $r(t) \in \mathbb{R}$ must be differentiated, and also consider that $w_{2}(t)=(d / d t) r(t)$ corresponds to its time derivative. Based on the assumption of $\left|\left(d^{2} / d t^{2}\right) r(t)\right| \leq r^{+}$, then the following auxiliary equations hold.

$$
\begin{aligned}
& \frac{d}{d t} w_{1}(t)=w_{2}(t) \\
& \frac{d}{d t} w_{2}(t)=\frac{d^{2}}{d t^{2}} r(t)
\end{aligned}
$$

The differential equations (44) correspond to the state representation of $r(t)$. The ST algorithm used to obtain the derivative of $r(t)$ satisfies the following.

$$
\begin{aligned}
\frac{d}{d t} \bar{w}_{1}(t) & =\bar{w}_{2}(t)-\lambda_{1}\left|\widetilde{w}_{1}(t)\right|^{1 / 2} \operatorname{sign}\left(\widetilde{w}_{1}(t)\right) \\
\frac{d}{d t} \bar{w}_{2}(t) & =-\lambda_{2} \operatorname{sign}\left(\widetilde{w}_{1}(t)\right) \\
\widetilde{w}_{1}=\bar{w}_{1}-w_{1} ; & \\
d(t) & =\frac{d}{d t} \bar{w}_{1}(t)
\end{aligned}
$$

Here $\bar{w}_{1}$ and $\bar{w}_{2}$ define the ST algorithm states and $\lambda_{1}, \lambda_{2}>0$ are the algorithm gains [56]. The variable $d(t)$ defines the differentiator result given in (45). Here,

$$
\operatorname{sign}(\nu)= \begin{cases}1 & \text { if } v>0 \\ -1 & \text { if } v<0\end{cases}
$$

Notice that the sign function used here agrees with the formulation given by [49] which is not defining the multivalued set for $\operatorname{sign}(0)$ corresponding to $[-1,+1]$. Notice that either definition yields the sliding mode equation without any ambiguousness.
The application of the STA as robust differentiator implies that the orthosis system (11) should be represented as the composition of the following couple of second order systems

$$
\begin{aligned}
\frac{d}{d t} \delta_{a, i}(t)= & \delta_{b, i}(t) \\
\frac{d}{d t} \delta_{b, i}(t)= & f_{i}\left(\delta_{a}(t), \delta_{b}(t)\right)+\sum_{j=1}^{2} g_{i j}\left(\delta_{a}(t)\right) u_{j}(t) \\
& +\eta_{i}\left(\delta_{a}(t), \delta_{b}(t), t\right)
\end{aligned}
$$

where $\delta_{a, i}$ and $\delta_{b, i}$ are the $i-t h$ and $(n+i)-t h$ states of (11), respectively. The nonlinear functions $f_{i}(\cdot)$ and $g_{i}(\cdot)$ are the functions associated with the states $\delta_{a, i}$ and $\delta_{b, i}$. Similarly, $\eta_{i}$ is the corresponding uncertainty to the same subsystem.

Using the STA, the impedance adaptive control only requires that the surface $S$ should be calculated as follows:

$$
S(t)=\delta_{a}+H \widehat{\delta}_{b}
$$

where $\widehat{\delta}_{b}$ is the estimated velocity based only on the information provided by $\delta_{a}$. The forward complete characteristic of the orthosis (based on its robotic manipulator configuration) allows substituting the original surface value $S$ by the estimated value using the output of the STA.

The study proposed in [51] provides the fundamentals for designing the soft-switch function between the human-incontrol and the robot-in-control operation modes. The mentioned study offers just the manner to propose the weighting function which gives a continuous variation between the human-in-control and the robot-in-control modes and vice versa. The rest of this study appears as a contribution in the sense that it offers a novel manner to consider the resistance effect of the patient against the orthosis action. Also, the explicit estimation of the control adaptive gains is given in this study considering the desired reference position. These adaptive gains also establish the gain restrictions which yield the design of a bounded feedback controller. Moreover, the introduction of an on-line robust and exact differentiator introduces the possibility of designing the output feedback variant of the suggested controller.

\section{The Orthosis Device}

A simplified orthosis prototype served to evaluate the suggested composite controller. The SolidWorks computer assisted design software verified the proposed mechanical design of the robotic orthosis. The design tools included in this software contributed to the selection of the construction material, the machining processes, and the actuators characteristics. A finite-element numerical analysis defined the static and dynamic characteristics of the cervical orthosis design.

The orthosis design included a support section aimed at carrying up the mobile section of the orthosis. This support structure consisted of an EVS-r4 collar, which is made of carbon fibers. The complete weight of this section was $0.635 \mathrm{~kg}$. The original objective of this collar was preventing whiplash-like injuries for sportsmen. 


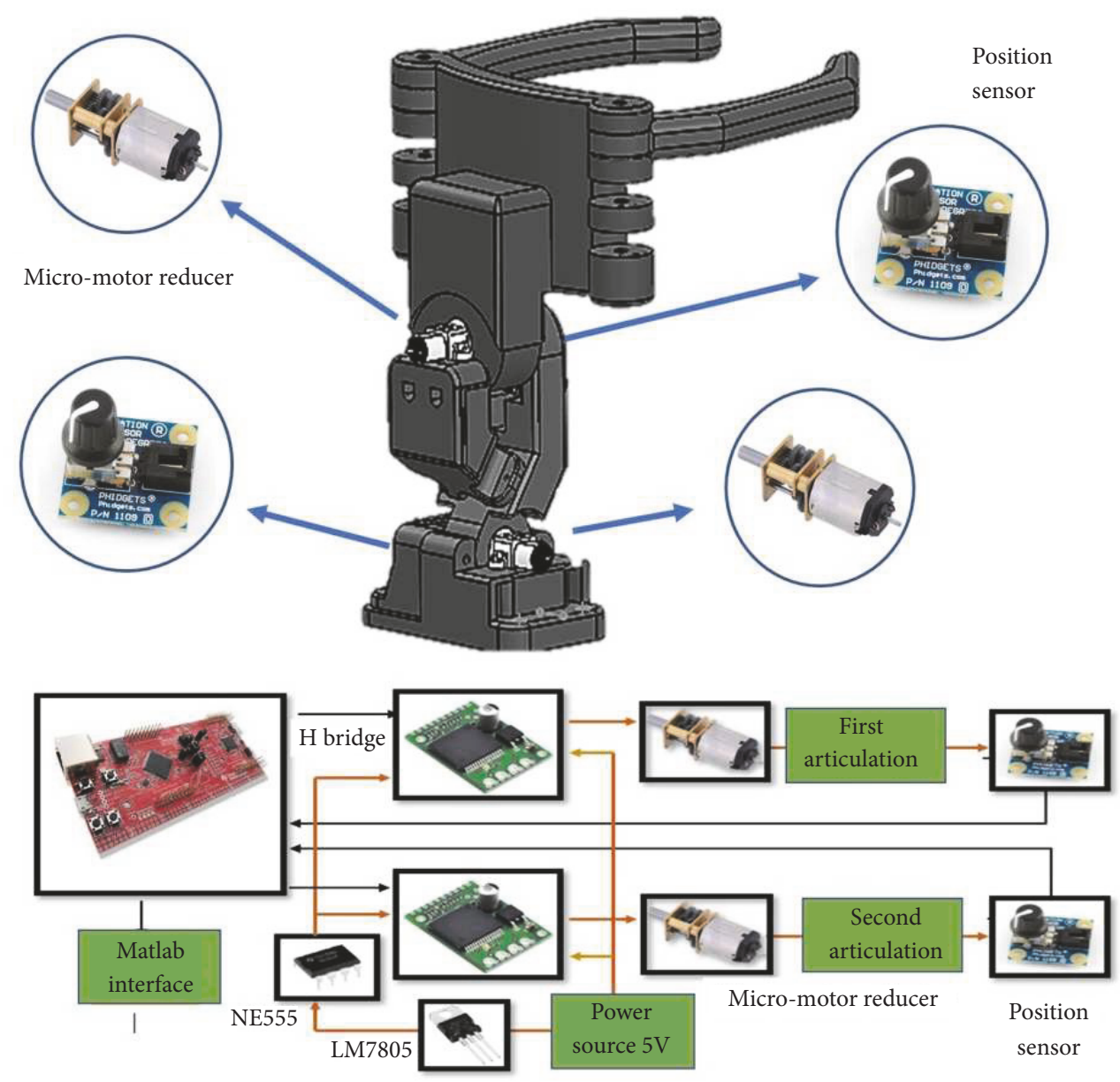

FIgURE 4: Cervical orthosis electronic instrumentation scheme depicting all the components needed to perform the proposed therapeutic procedures. The rotatory potentiometers are used to measure the angular position at each of the two articulations. The $\mathrm{H}$-bridge electronic boards as well as the micro DC motors are the electronic devices enforcing the movement of each orthosis coordinate. The optical coupling devices isolated the high-power phase of the digital micro-controller (Tiva C 1294 Series).

A three-dimensional printing technique constructed the mobile section of the orthosis. The polymer acrylonitrile butadiene styrene was the building material for the orthosis. A $Z-18$ replicator printing machine executed the diverse machining processes in the orthosis device. The printing characteristics were as follows: resolution $=0.02 \mathrm{~mm}$, velocity $=1 \mathrm{~cm} / \mathrm{min}$, etc.

The instrumentation of the orthosis and its integration with the mechanical design and other steps are represented in Figure 4.

The selection of each joint actuator considered the torque in the corresponding junction; a set of two DC motors was selected. An electromechanical analysis was realized, in order to obtain the torque corresponding to each joint of the orthosis device.

The set of sensors was composed of five linear precision single turn potentiometers used for measuring the position for each joint. The maximum resistance of the potentiometer was $10 \mathrm{k} \Omega$ and it is designed to be $3.3 \mathrm{~V}$ compatible; the minimum range is $0.3^{\circ}$ (Phidgets Slider 60, USA). The actuators were DC motors (250:1 HPCB 12V, Pololu, USA) with $10.5 \mathrm{~g}$ weight, high-power carbon brushes, operation voltage $=12$ volts, angular velocity of $130 \mathrm{RPM}$, torque $=3.0$ Kg.cm. The driving of actuators implemented the VNH5019 (Pololu EVAL-VNH5019-P2, USA) Motor Driver Carrier (motor driver IC working with a voltage range of 5.5 to 24 $\mathrm{V}$, continuous current of $12 \mathrm{~A}, 2.5$ to $5 \mathrm{~V}$ logic levels, input $20 \mathrm{kHz}$ of PWM). The current sensor at the input of the actuator was the 20Amp Current Sensor AC/DC with 5000.0 $\mathrm{v}$ isolation (Phidgets RB-Phi-72, USA).

To implement the control algorithm, a low-cost development platform was used. This platform works with a high speed Texas Instrument ${ }^{\circledR}$ ARM $^{\circledR}$ Cortex-M4F microcontroller. The platform is the Tiva C Series TM4C1294 ${ }^{\circledR}$ and has the following features: $120 \mathrm{MHz}, 32$ bits CPU, 1 MB memory flash, $256 \mathrm{~KB}$ SRAM, 6KB EEPROM, and serial communication interfaces. The analog-digital converter (ADC) channels read the angular position sensor signals. The signals are processed individually. Because the movements of all other joints are considered as perturbations, 

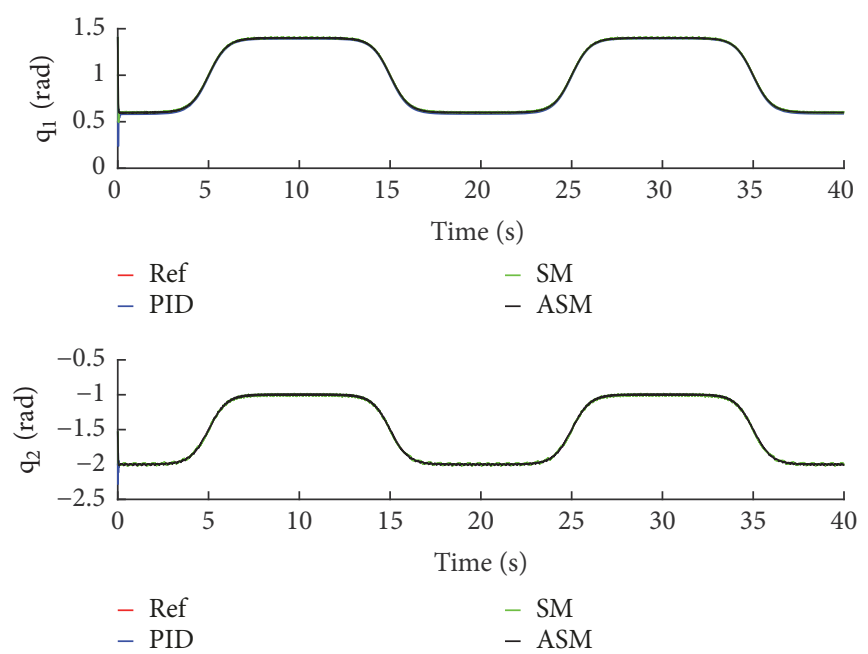

Figure 5: Comparison of the tracking trajectories associated with the movements executed by the simulated orthosis regulated by the proposed impedance adaptive sliding mode controller, the PID, and the regular first-order sliding mode variant. The simulation was realized in the Matlab/Simulink environment using the SimMechanics Toolbox.

this led to the development of a novel scheme to manipulate each of the movable sections of the orthosis; the derivative and the control for each actuator are computed. Output signals are sent through the general purpose input-output (GPIO) pins as pulse-width modulation (PWM) signals. The output signals of the platform pass through an optocoupler to isolate the power stage and the controller board. The power stage for the actuators was handled by an $\mathrm{H}$-bridge board; in the case of the micro metal gear motors, a VNH5019 ${ }^{\circledR}$ device from Pololu ${ }^{\circledR}$ was used. This device is a dual $\mathrm{H}$-bridge and it allows two motors to be driven at the same time; for the other three metal gear motors, a Pair Motor Driver Carrier VNH3SP $30^{\circledR}$ of Pololu ${ }^{\circledR}$ was used. These drivers support the technical specification for the actuators estimated for the orthosis operation.

\section{Simulated Evaluation of the Suggested Controller}

The first part of results corresponded to the class of exercises developed by the AO. These numerical evaluations were selected according to the regular treatment methods used in actual physiotherapies of the whiplash syndrome. These sequences of movements were included in Figure 5 and they correspond to some exercises moving both articulations together. These movements were obtained as solution of the numerical implementation (using the so-called SimMechanics Toolbox) of the AO in Matlab. This orthosis was simulated to evaluate the performance of the distributed strategy of adaptive control. The simulated model in Matlab was evaluated with the actual mass and dimensions for each mechanical section included in the AO. This strategy served to evaluate the controller and define the gains that must be used for tuning both differentiators as well as controllers.

The trajectories shown in Figure 5 correspond to the simultaneous lateral and frontal movements of the neck. In this case, the study evaluated the tracking of the reference trajectories, but considering that the patient is not offering any resistance to the orthosis action.

Figure 5 shows the comparison of trajectories obtained by the application of three different controllers: PID (blue line), first-order (green line), and the adaptive sliding mode (black line) variants. The PID controller structure satisfied the following.

$$
\tau(t)=K_{p} \delta_{a}(t)+K_{d} \delta_{b}(t)+K_{i} \int_{s=0}^{t} \delta_{a}(s) d s
$$

The simulation of this controller used the following gains.

$$
\begin{aligned}
K_{p} & =\left[\begin{array}{cc}
50.0 & 0.0 \\
0.0 & 50.0
\end{array}\right] \\
K_{d} & =\left[\begin{array}{ll}
2.0 & 0.0 \\
0.0 & 2.0
\end{array}\right] \\
K_{i} & =\left[\begin{array}{ll}
0.5 & 0.0 \\
0.0 & 0.5
\end{array}\right]
\end{aligned}
$$

The second controller used for comparison purposes was a first-order sliding mode variant satisfying

$$
\tau(t)=K_{S M}\left(\delta_{a}(t)+H \delta_{b}(t)\right)
$$

with $K_{S M}$ being the gain of this controller and $H$ the positive definite matrix given by the following.

$$
\begin{aligned}
H & =\left[\begin{array}{cc}
0.01 & 0.0 \\
0.0 & 0.01
\end{array}\right] \\
K_{S M} & =\left[\begin{array}{cc}
40.0 & 0.0 \\
0.0 & 40.0
\end{array}\right]
\end{aligned}
$$



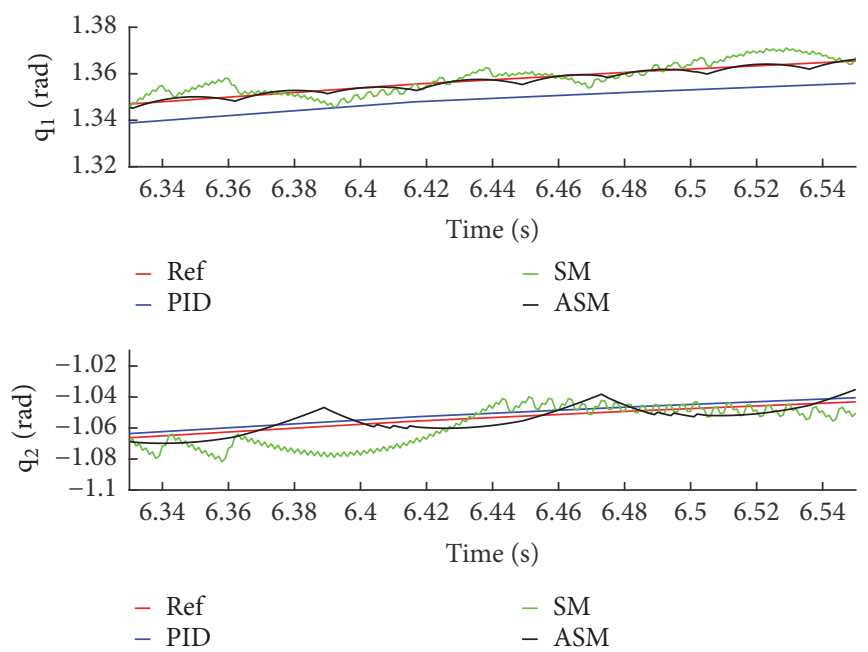

Figure 6: Amplified view of the trajectories obtained with the implementation of the different controllers corresponding to reference trajectories describing movements executed by the simulated orthosis. The simulation was realized in the Matlab/Simulink environment using the SimMechanics Toolbox.

The proposed adaptive controller (22) was simulated using the following parameters.

$$
\begin{aligned}
K_{t r}(0) & =\left[\begin{array}{cc}
50.0 & 0.0 \\
0.0 & 55.0
\end{array}\right] \\
K_{c}(0) & =\left[\begin{array}{cc}
20.0 & 0.0 \\
0.0 & 25.0
\end{array}\right] \\
M & =\left[\begin{array}{cc}
45.0 & 0.0 \\
0.0 & 20.0
\end{array}\right] \\
\epsilon & =0.05 \\
\lambda & =0.20 \\
\gamma_{0} & =2.5 \\
\gamma_{1} & =4.2
\end{aligned}
$$

A set of diverse reference trajectories evaluated the ability of the cervical orthosis to realize the movements corresponding to distinct therapies. These different trajectories were developed by implementing the composite sigmoid functions. Figure 5 demonstrates the corresponding movements which were produced by the controller in simulation at different stages of the therapy. This figure emphasizes the effect of tracking controller on both articulations leading to the general movement of the orthosis. Notice that in this figure, the generated trajectories of the three controllers seem to be similar. Nevertheless, one may notice that there are significant differences between them. Figure 6 demonstrates the trajectories produced by all the three evaluated controllers in a time range of 6.33-6.54 seconds. This amplified view exhibits the improvements in the tracking task of the adaptive controller (without taking into account the effect of the patient resistance). The adaptive gains included in the controller reduced the regular high-frequency oscillations produced as a consequence of introducing the traditional discontinuous sliding mode controller. Moreover, the proposed controller tracked the reference trajectory with smaller tracking error than the other two controllers. This characteristic was observed for both articulations in the cervical orthosis.

The control signals obtained in the numerical evaluation of trajectory tracking for the cervical orthosis demonstrated that the adaptive sliding controller proposed in this study reduced the energy needed to complete the reference track (Figure 7). The behavior of the control action demonstrates that less energy is needed to complete the trajectory problem than that in the case when PID or the nonadaptive sliding mode controllers were considered. This can be confirmed by the range where the control action evolves during the numerical simulations. Moreover, the adaptive controller showed a similar response to the PID with better tracking quality, and it has smaller high-frequency oscillations than the regular sliding mode version.

The diminution in the vibrations exhibited in the control signals plays a relevant role in the orthosis-human interaction. These vibrations may cause long-term injuries on either the vertebra or the muscles needed to mobilize the patient's cervical section. This aspect represents a serious issue in the application of active orthosis controlled by sliding mode controller. Nevertheless, the accurate gain tuning based on the adaptive form as well as the force compensation reduced the oscillations on the tracking trajectory as demonstrated in this study.

The second set of numerical evaluations consisted in describing the effect of the external force $f_{\text {ext }}$ and how it modifies the trajectories produced by the orthosis. Figure 8 demonstrates the components ( $\mathrm{a}$ and $\mathrm{b}$ ) of the simulated external force, which represents the patient resistance to the orthosis action. These components correspond to gradual and fast patient's resistance which is maintained for 5 seconds 


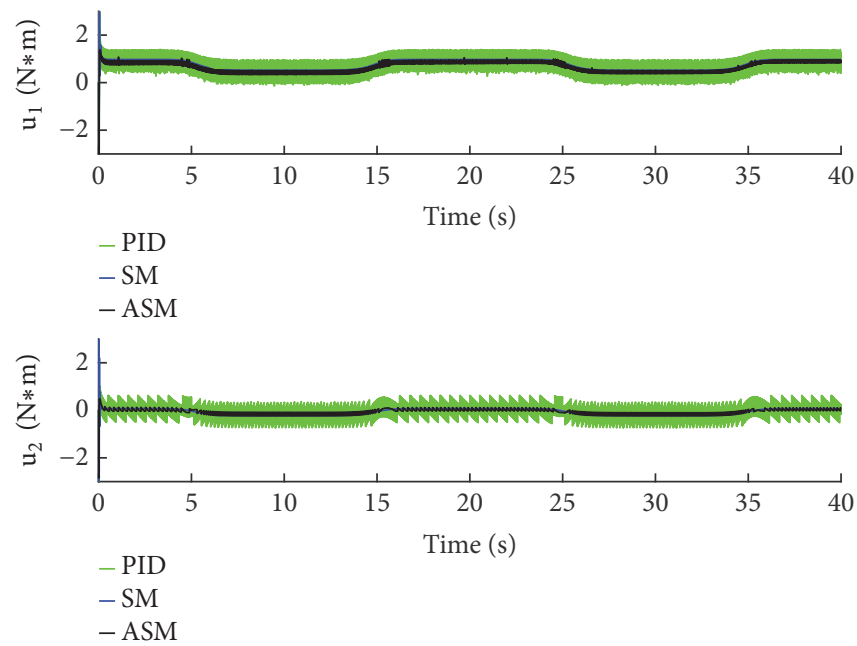

Figure 7: Comparison of the time evolution for the control signals associated with the movements executed by the simulated orthosis regulated by the PID and the classical first-order and the impedance adaptive sliding mode controller.

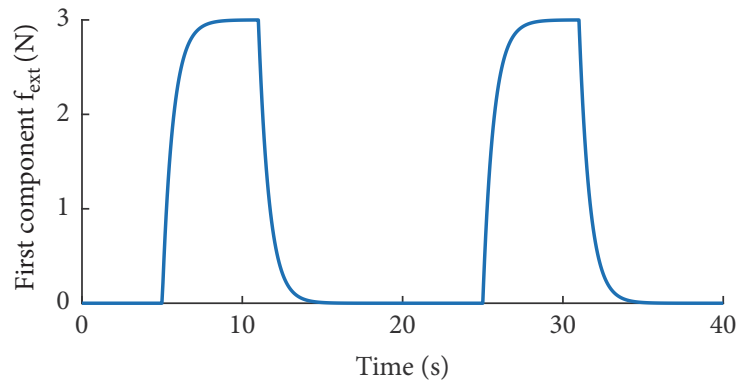

(a)

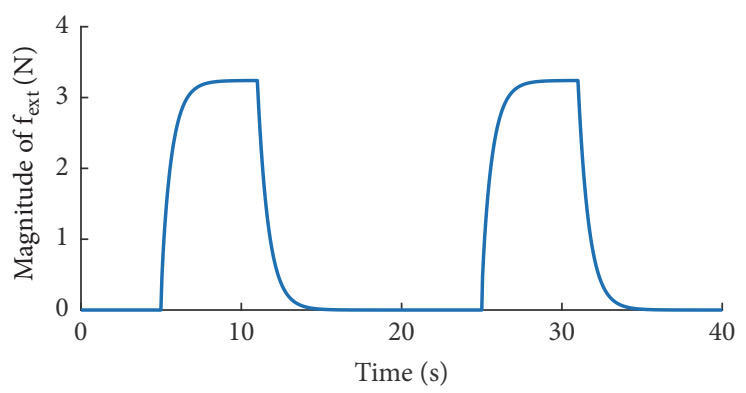

(c)

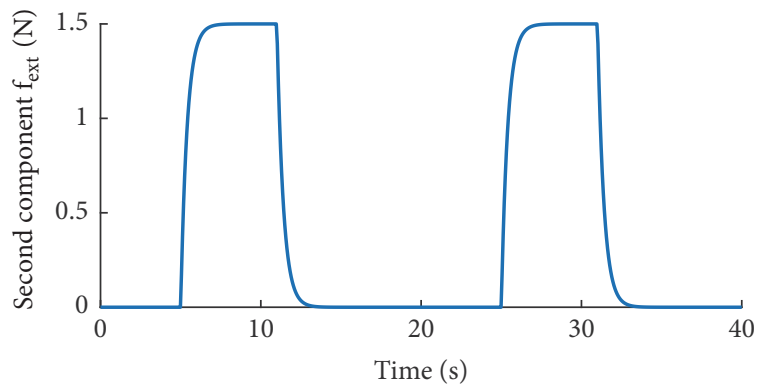

(b)

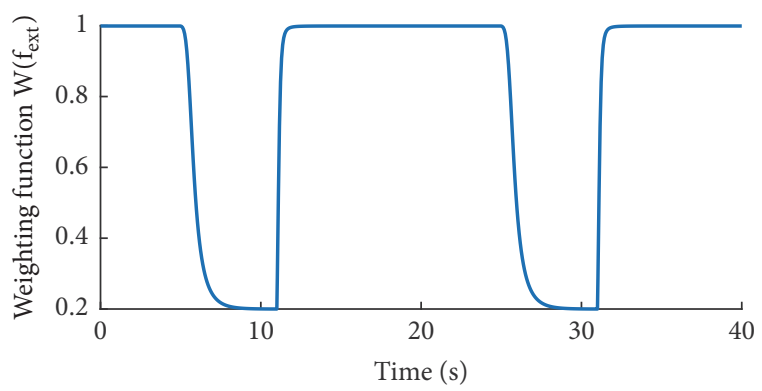

(d)

Figure 8: Characterization of the simulated external force $f_{\text {ext }}$ used in the numerical evaluation of the impedance adaptive sliding mode controller. (a) First component of $f_{\text {ext }}$, (b) second component of $f_{\text {ext }}$, (c) magnitude of $f_{\text {ext }}$, and (d) evaluation of the weighing function in terms of the simulated $f_{\text {ext }}$.

and then released. The magnitude of this external force is depicted in Figure 8(c). The time variation of this magnitude evaluated on the weighting function $w\left(f_{\text {ext }}\right)$ appears in Figure $8(\mathrm{~d})$. The variation of this weighting defines the relative contribution of both sections of the controller, $\tau_{t r}$ and $\tau_{c}$.

The presence of the external force $f_{\text {ext }}$ modifies the trajectory tracking of the reference signal in the cervical orthosis. Notice that if the external force produces the modification of the weighting function, and then the tracking quality is reduced leading to the loss of the tracking as noticed in Figure 9. Once the external force disappears, then the controllers enforce the tracking of the reference trajectory. This fact, confirmed in two cases as shown in Figure 9, demonstrates the benefits of introducing the weighted controller proposed in this study.

Notice that Figure 9 contains the comparison of the adaptive sliding mode without and with the impedance evaluation effect. This comparison demonstrates that if the impedance effect is not considered, the adaptive sliding mode 


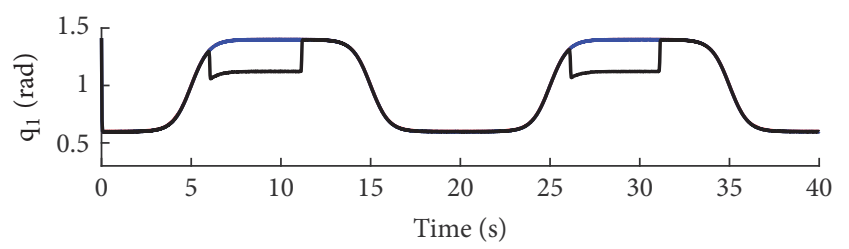

$$
\begin{aligned}
& \text { - PID } \\
& \text { - ASM } \\
& \text { - ASM-Impedance }
\end{aligned}
$$

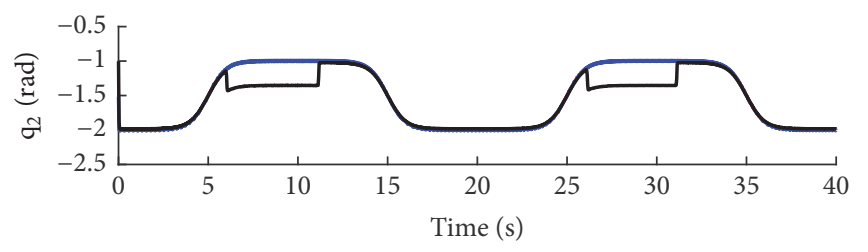

- PID

$-\mathrm{ASM}$

- ASM-Impedance

FIGURE 9: Comparison of the trajectory tracking associated with the movements executed by the simulated orthosis regulated by the proposed impedance adaptive sliding mode controller with and without taking the patient resistance effect.

controller keeps tracking the reference without considering the resistance of the patient to the orthosis action. This is a remarkable advance of the suggested controller because it may ensure a safe operation of the patient-orthosis process for the rehabilitation of the whiplash syndrome. Moreover, the resistance of the patient to the orthosis ensures that the orthosis keeps a constant position (shown in the figure at the same time when the external force appears). This condition ensures that the patient is ruling the orthosis activity, and the tracking problem is not considered in this case.

\section{Experimental Results}

The functioning evaluation of the actual cervical orthosis used the adaptive controller implemented in the TIVA1294 device. The explicit discretization algorithm defined the implementation of the adaptive controller. The controller used the Code Composer v8.0 software.

The numerical integration implemented in the TIVA129 board was the first-order Euler ODE algorithm. The predefined sampling period was $0.01 \mathrm{~s}$ (this value was confirmed experimentally).

The experimental evaluation of the orthosis function considered a dummy phantom to characterize the tracking trajectory effectiveness. The dummy cannot move freely because it is fixed on a supporting spring, which is representing the force exerted by the neck muscles. Then, the force applied by the spring on the robot could be regulated by holding the spring tight to the base. In this way, the dummy served to prove the efficiency of the suggested controller. Moreover, the modification of the holding force between the basement and the spring represents different scenarios of impedance responses between the patient and the proposed orthosis. An extended polystyrene head-like dummy phantom attached to the constructed orthosis served as experimental platform. The neck resistance and elasticity were represented by a metallic spring (Hooke constant of $4.8 \mathrm{~N} / \mathrm{m}$ ), which was attached to the bottom part of the headlike phantom (Figure 10).

The implemented impedance adaptive sliding mode controller used the following gains to adjust the orthosis movement.

$$
\begin{aligned}
K_{t r}(0) & =\left[\begin{array}{ll}
15 & 0.0 \\
0.0 & 25
\end{array}\right] \\
K_{c}(0) & =\left[\begin{array}{cc}
12 & 0.0 \\
0.0 & 9
\end{array}\right] \\
M & =\left[\begin{array}{ll}
12 & 0.0 \\
0.0 & 12
\end{array}\right] \\
\epsilon & =0.05 \\
\lambda & =0.20 \\
\gamma_{0} & =2.5 \\
\gamma_{1} & =2.2
\end{aligned}
$$

Figure 11 depicts the trajectories of the measured angles from the potentiometric sensors placed at each articulation in the orthosis. The reference angular movement corresponded to a basic lateral movement, which is considered one of the first therapies in the rehabilitation of the whiplash syndrome. The selected reference trajectories for each articulation confirmed the capacity of the designed cervical orthosis to realize the proposed movements corresponding to a simple lateral oscillatory movement, one of the first therapy proposed by specialists. Notice that the suggested experimental result has the presence of the resistance enforced by the spring 


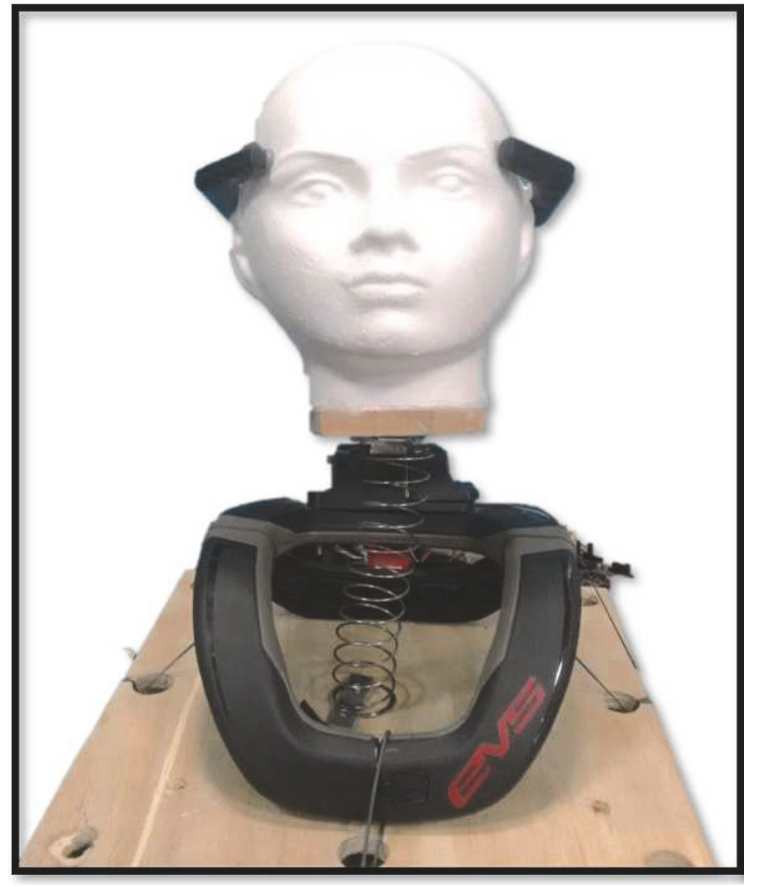

(a) Frontal view

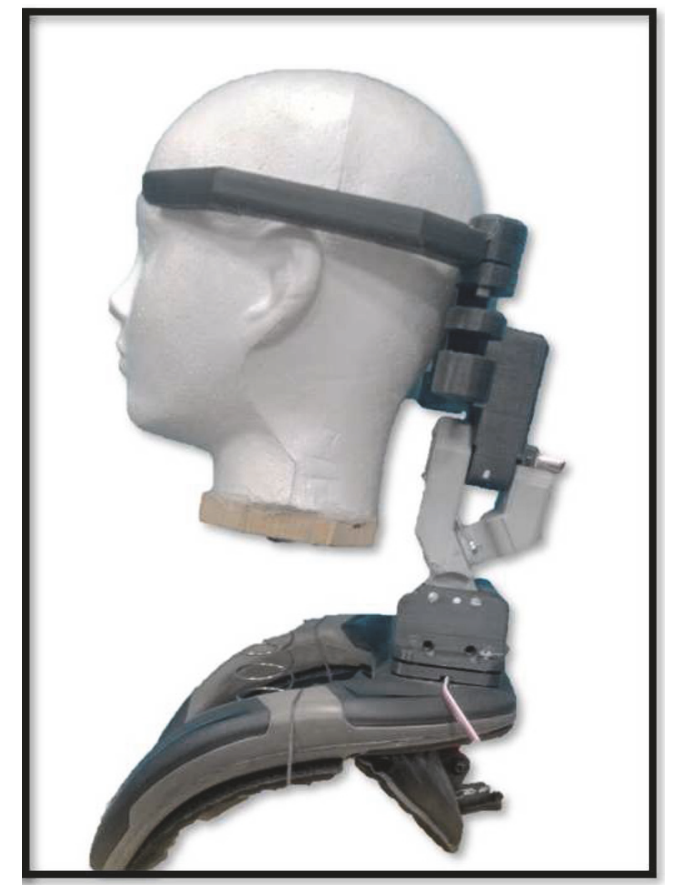

(b) Lateral view

FIGURE 10: Frontal and lateral projections of the developed cervical orthosis and the attached head-like dummy phantom. This phantom has the spring attached at the bottom section, which represented the neck resistance and elasticity.

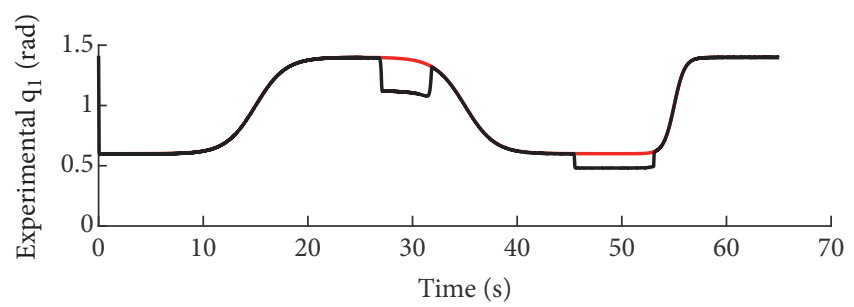

- Ref Exp

- ASM-Impedance

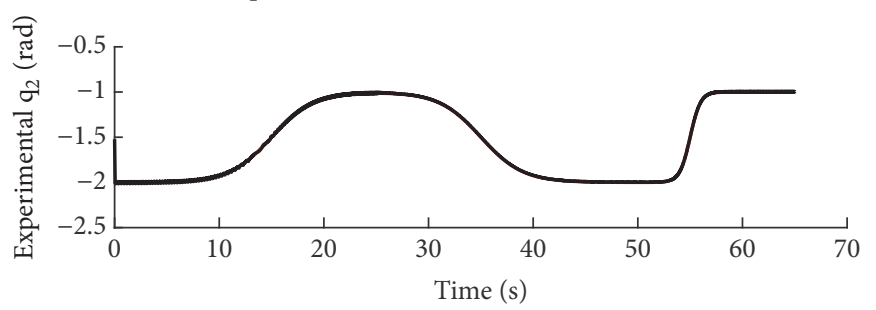

- Ref Exp

- ASM-Impedance

FIGURE 11: Experimental tracking trajectories associated with the movements executed by the real orthosis regulated by the proposed impedance adaptive sliding mode controller.

device. This is the reason of the deviation observed along the intervals of $[28.4,31.5] s$ and $[46.3,54.5] s$. Such experimental results confirmed the effectiveness of the weighted controller suggested in this study.
Figure 12 shows a sequence of photos taken along the proposed trajectory shown in Figure 11. The reference trajectories were proposed to mobilize the head in the lateral plane. The orthosis movements were produced by the 

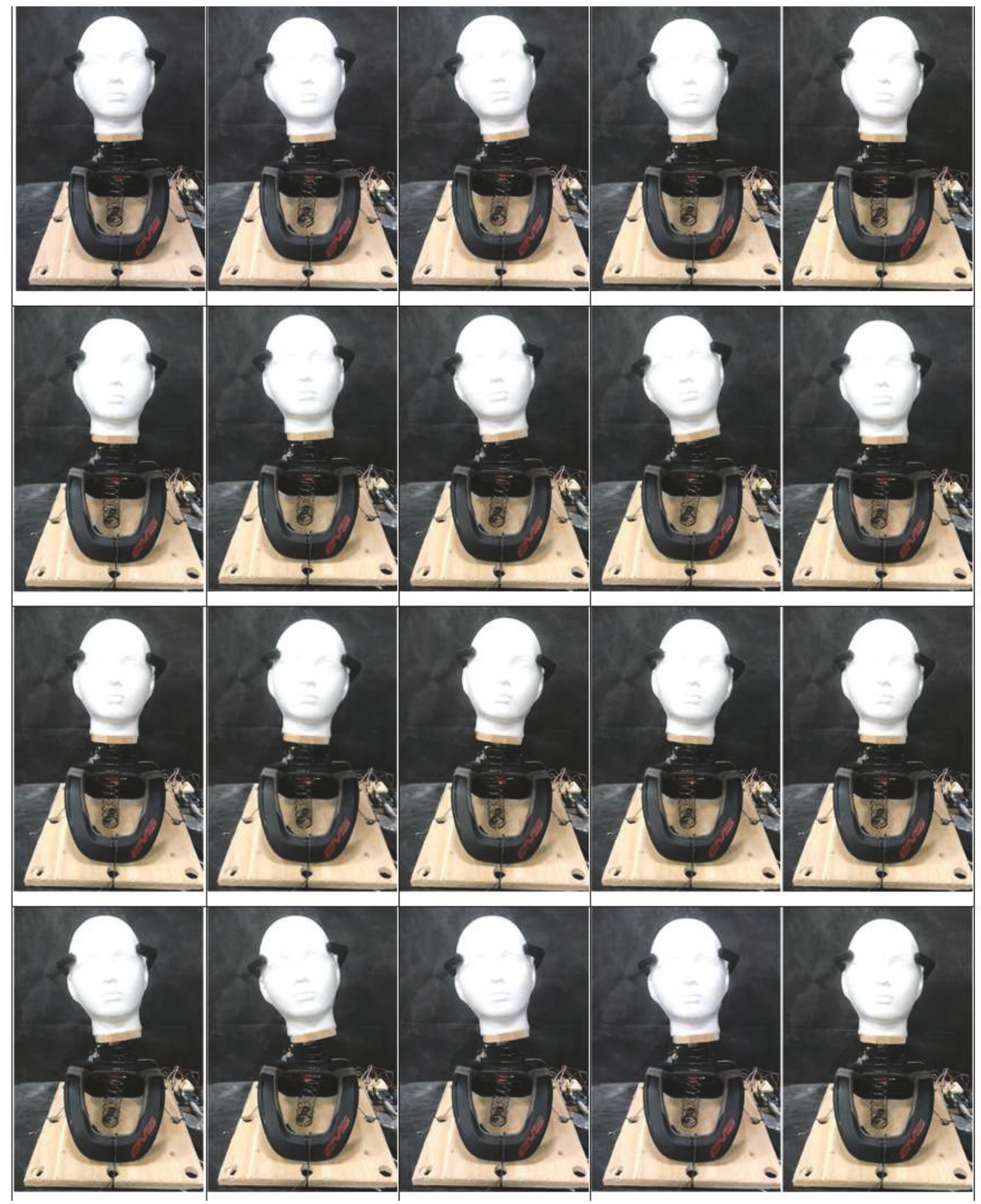

FIGURE 12: Sequence of photos showing the movements executed by the real orthosis regulated by the proposed impedance adaptive sliding mode controller considering the resistance of the spring attached to the head-like dummy phantom.

controller and tracked the proposed trajectory despite the presence of the resistance induced by the spring. Similar evaluations were realized for three different trajectories where individual articulations or both articulations, simultaneously, were mobilized.

\section{Conclusions}

This study detailed the design, numerical evaluation, and experimental validation of an impedance adaptive sliding mode controller aimed at regulating the movements of an 
active cervical orthosis. The controller considered the effect of the patient resistance to the orthosis action as well as the trajectory tracking problem considering the design of reference trajectories corresponding to regular therapy sequence of movements. A novel weighting function introduces a dynamic rule to characterize the activity of both controllers, the tracking oriented one, and the force dependent realization. The specific rules to select the parameters of the adaptive controller were proposed. The numerical evaluations of the controller confirmed the superior performance of the proposed controller with respect to the classical PID and firstorder sliding mode variant. Additionally, the weighting rule for the components (tracking and impedance) was evaluated in the case when the patient resisted the orthosis action. The controller responded avoiding the mobilization of the cervical section of the patient if the resistance is present. The suggested controller did not have the usual switched structure proposed in diverse impedance base control designs, which simplified the theoretical analysis of the trajectory tracking error convergence. The suggested controller, evaluated on an own-developed cervical orthosis, was able to complete the tracking trajectory corresponding to a basic cervical rehabilitation therapy suggested for patients with whiplash syndrome. This study considered the application of a sliding mode controller with state dependent gains which provided a solution for a robotic orthosis. Notice that novel methods including parametric and nonparametric adaptive estimations of uncertainties and perturbations may offer a distinctive manner of solving the tracking trajectory problem for rehabilitation devices such as the one considered in this study $[38,58,59]$.

\section{Data Availability}

The simulation and experimental data used to support the findings of this study are available from the corresponding author upon request.

\section{Conflicts of Interest}

The authors declare that they have no conflicts of interest.

\section{References}

[1] J. M. Matuszak, J. McVige, J. McPherson, B. Willer, and J. Leddy, "A practical concussion physical examination toolbox: evidence-based physical examination for concussion," Sports Health, vol. 8, no. 3, pp. 260-269, 2015.

[2] D. C. Tong and J. Breeze, "Damage control surgery and combatrelated maxillofacial and cervical injuries: A systematic review," British Journal of Oral and Maxillofacial Surgery, vol. 54, no. 1, pp. 8-12, 2016.

[3] R. Kanwar, B. E. Delasobera, K. Hudson, and W. Frohna, "Emergency department evaluation and treatment of cervical spine injuries," Emergency Medicine Clinics of North America, vol. 33, no. 2, pp. 241-282, 2015.

[4] P. Huang, A. Anissipour, W. McGee, and L. Lemak, "Returnto-play recommendations after cervical, thoracic, and lumbar spine injuries: a comprehensive review," Sports Health, vol. 8, no. 1, pp. 19-25, 2016.
[5] N. Khezri, T. Ailon, and B. K. Kwon, "Treatment of facet injuries in the cervical spine," Neurosurgery Clinics of North America, vol. 28, no. 1, pp. 125-137, 2017.

[6] C. Hrysomallis, "Neck muscular strength, training, performance and sport injury risk: a review," Sports Medicine, vol. 46, no. 8, pp. 1111-1124, 2016.

[7] A. Cesarani, D. Alpini, R. Boniver et al., Whiplash Injuries: Diagnosis and Treatment, Springer Science \& Business Media, 2013.

[8] J. J. Wong, P. Côté, H. M. Shearer et al., "Clinical practice guidelines for the management of conditions related to traffic collisions: A systematic review by the OPTIMa Collaboration," Disability and Rehabilitation, vol. 37, no. 6, pp. 471-489, 2015.

[9] C. Ritchie, J. Hendrikz, G. Jull, J. Elliott, and M. Sterling, "External validation of a clinical prediction rule to predict full recovery and ongoing moderate/severe disability following acute whiplash injury," Journal of Orthopaedic \& Sports Physical Therapy, vol. 45, no. 4, pp. 242-250, 2015.

[10] P. Sarrami, E. Armstrong, J. M. Naylor, and I. A. Harris, "Factors predicting outcome in whiplash injury: a systematic meta-review of prognostic factors," Journal of Orthopaedics and Traumatology, vol. 18, no. 1, pp. 9-16, 2017.

[11] K. Nas, L. Yazmalar, V. Şah, A. Aydin, and K. Öneş, "Rehabilitation of spinal cord injuries," World Journal of Orthopedics, vol. 6, no. 1, pp. 8-16, 2015.

[12] S. Mehta, A. McIntyre, S. Janzen, E. Loh, and R. Teasell, "Systematic review of pharmacologic treatments of pain after spinal cord injury: an update," Archives of Physical Medicine and Rehabilitation, vol. 97, no. 8, pp. 1381-1391, 2016.

[13] J. J. Leddy, J. G. Baker, and B. Willer, "Active rehabilitation of concussion and post-concussion syndrome," Physical Medicine and Rehabilitation Clinics of North America, vol. 27, no. 2, pp. 437-454, 2016.

[14] G. E. Francisco, N. Yozbatiran, J. Berliner et al., "Robot-assisted training of arm and hand movement shows functional improvements for incomplete cervical spinal cord injury," American journal of physical medicine \& rehabilitation, vol. 96, no. 10, pp. S171-S177, 2017.

[15] A. S. Burns, R. J. Marino, S. Kalsi-Ryan et al., "Type and timing of rehabilitation following acute and subacute spinal cord injury: a systematic review," Global Spine Journal, vol. 7, no. 3, pp. 175-194, 2017.

[16] C. Thompson, J. F. Gonsalves, and D. Welsh, "Hyperextension injury of the cervical spine with central cord syndrome," European Spine Journal, vol. 24, no. 1, pp. 195-202, 2014.

[17] S. Mateo, A. Roby-Brami, K. T. Reilly, Y. Rossetti, C. Collet, and G. Rode, "Upper limb kinematics after cervical spinal cord injury: a review," Journal of NeuroEngineering and Rehabilitation, vol. 12, no. 1, 9 pages, 2015.

[18] K. Barati, M. Arazpour, R. Vameghi, A. Abdoli, and F. Farmani, "The effect of soft and rigid cervical collars on head and neck immobilization in healthy subjects," Asian Spine Journal, vol. 11, no. 3, pp. 390-395, 2017.

[19] L. Rogers, "No place for the rigid cervical collar in pre-hospital care," International Paramedic Practice, vol. 7, no. 1, pp. 12-15, 2017.

[20] M. C. Makhni, E. C. Makhni, E. F. Swart, and C. S. Day, "Initial cervical spine management," in Orthopedic Emergencies, pp. 79, Springer International Publishing, 2017.

[21] A. F. Burnett, F. L. Naumann, R. S. Price, and R. H. Sanders, "A comparison of training methods to increase neck muscle strength," Work, vol. 25, no. 3, pp. 205-210, 2005. 
[22] P. C. Ivancic and C. J. Telles, "Neck motion due to the halovest in prone and supine positions," in Proceedings of the 6th World Congress of Biomechanics (WCB '10), vol. 31 of IFMBE Proceedings, pp. 569-572, Springer, Berlin, Germany, 2010.

[23] T. M. Gavin, G. Carandang, R. Havey, P. Flanagan, A. Ghanayem, and A. G. Patwardhan, "Biomechanical analysis of cervical orthoses in flexion and extension: A comparison of cervical collars and cervical thoracic orthoses," Journal of Rehabilitation Research and Development, vol. 40, no. 6, pp. 527-537, 2003.

[24] R. Jiménez-Fabián and O. Verlinden, "Review of control algorithms for robotic ankle systems in lower-limb orthoses, prostheses, and exoskeletons," Medical Engineering \& Physics, vol. 34, no. 4, pp. 397-408, 2012.

[25] J. F. Cusick, F. A. Pintar, and N. Yoganandan, "Whiplash syndrome: Kinematic factors influencing pain patterns," The Spine Journal, vol. 26, no. 11, pp. 1252-1258, 2001.

[26] R. Ferrari and H. Schrader, "The late whiplash syndrome: a biopsychosocial approach," Journal of Neurology, Neurosurgery \& Psychiatry, vol. 70, no. 6, pp. 722-726, 2001.

[27] M. Koelbaek Johansen, T. Graven-Nielsen, A. Schou Olesen, and L. Arendt-Nielsen, "Generalised muscular hyperalgesia in chronic whiplash syndrome," Pain, vol. 83, no. 2, pp. 229-234, 1999.

[28] L. Provinciali, M. Baroni, L. Illuminati, and M. G. Ceravolo, "Multimodal treatment to prevent the late whiplash syndrome," Journal of Rehabilitation Medicine, vol. 28, no. 2, pp. 105-111, 1996.

[29] M. Sterling, "Treatment of patients with whiplash associated disorders," Psychological Approaches to Pain Management: A Practitioner's Handbook, p. 425, 2018.

[30] A. J. del-Ama, A. D. Koutsou, J. C. Moreno, A. de-los-Reyes, Á. Gil-Agudo, and J. L. Pons, "Review of hybrid exoskeletons to restore gait following spinal cord injury," Journal of Rehabilitation Research and Development, vol. 49, no. 4, pp. 497-514, 2012.

[31] M. R. Tucker, J. Olivier, A. Pagel et al., "Control strategies for active lower extremity prosthetics and orthotics: A review," Journal of NeuroEngineering and Rehabilitation, vol. 12, no. 1, 2015.

[32] L. Marchal-Crespo and D. J. Reinkensmeyer, "Review of control strategies for robotic movement training after neurologic injury," Journal of NeuroEngineering and Rehabilitation, vol. 6, no. 1, article 20, 2009.

[33] X. Li, Y. Pan, G. Chen, and H. Yu, "Multi-modal control scheme for rehabilitation robotic exoskeletons," International Journal of Robotics Research, vol. 36, no. 5-7, pp. 759-777, 2017.

[34] H. Yu, S. Huang, G. Chen, Y. Pan, and Z. Guo, "Human-robot interaction control of rehabilitation robots with series elastic actuators," IEEE Transactions on Robotics, vol. 31, no. 5, pp. 1089-1100, 2015.

[35] J. Zhang and C. C. Cheah, "Passivity and stability of humanrobot interaction control for upper-limb rehabilitation robots," IEEE Transactions on Robotics, vol. 31, no. 2, pp. 233-245, 2015.

[36] M.-S. Ju, C.-C. K. Lin, D.-H. Lin, I.-S. Hwang, and S.-M. Chen, "A rehabilitation robot with force-position hybrid fuzzy controller: Hybrid fuzzy control of rehabilitation robot," IEEE Transactions on Neural Systems and Rehabilitation Engineering, vol. 13, no. 3, pp. 349-358, 2005.

[37] W. He, S. S. Ge, Y. Li, E. Chew, and Y. S. Ng, "Neural network control of a rehabilitation robot by state and output feedback," Journal of Intelligent \& Robotic Systems, vol. 80, no. 1, pp. 15-31, 2015.
[38] K. Guo, Y. Pan, and H. Yu, "Composite learning robot control with friction compensation: a neural network-based approach," IEEE Transactions on Industrial Electronics, 2018.

[39] M. Bernhardt, M. Frey, G. Colombo, and R. Riener, "Hybrid force-position control yields cooperative behaviour of the rehabilitation robot lokomat," in Proceedings of the 9th International Conference on Rehabilitation Robotics, ICORR '05, pp. 536-539, IEEE, Chicago, Ill, USA, 2005.

[40] P. K. Jamwal, S. Hussain, M. H. Ghayesh, and S. V. Rogozina, "Impedance control of an intrinsically compliant parallel ankle rehabilitation robot," IEEE Transactions on Industrial Electronics, vol. 63, no. 6, pp. 3638-3647, 2016.

[41] W. Meng, Q. Liu, Z. Zhou, Q. Ai, B. Sheng, and S. S. Xie, "Recent development of mechanisms and control strategies for robotassisted lower limb rehabilitation," Mechatronics, vol. 31, pp. 132-145, 2015.

[42] P. Song, Y. Yu, and X. Zhang, "A tutorial survey and comparison of impedance control on robotic manipulation," Robotica, vol. 37, no. 5, pp. 801-836, 2019.

[43] J. A. Blaya and H. Herr, "Adaptive control of a variableimpedance ankle-foot orthosis to assist drop-foot gait," IEEE Transactions on Neural Systems and Rehabilitation Engineering, vol. 12, no. 1, pp. 24-31, 2004.

[44] S. Hussain, S. Q. Xie, and P. K. Jamwal, "Adaptive impedance control of a robotic orthosis for gait rehabilitation," IEEE Transactions on Cybernetics, vol. 43, no. 3, pp. 1025-1034, 2013.

[45] I. Ranatunga, F. L. Lewis, D. O. Popa, and S. M. Tousif, "Adaptive admittance control for human-robot interaction using model reference design and adaptive inverse filtering," IEEE Transactions on Control Systems Technology, vol. 25, no. 1, pp. 278-285, 2017.

[46] M. Sharifi, S. Behzadipour, and G. Vossoughi, "Nonlinear model reference adaptive impedance control for human-robot interactions," Control Engineering Practice, vol. 32, pp. 9-27, 2014.

[47] H. Modares, I. Ranatunga, F. L. Lewis, and D. O. Popa, “Optimized assistive human-robot interaction using reinforcement learning," IEEE Transactions on Cybernetics, vol. 46, no. 3, pp. 655-667, 2016.

[48] V. I. Utkin and A. S. Poznyak, "Adaptive sliding mode control with application to super-twist algorithm: equivalent control method," Automatica, vol. 49, no. 1, pp. 39-47, 2013.

[49] V. Utkin, J. Guldner, and J. Shi, Sliding Mode Control in ElectroMechanical Systems, Automation and Control Engineering, 2009.

[50] C. C. Cheah, S. Kawamura, and S. Arimoto, "Feedback control for robotic manipulator with an uncertain jacobian matrix," Journal of Robotic Systems, vol. 16, no. 2, pp. 119-134, 1999.

[51] X. Li, Y. Pan, G. Chen, and H. Yu, "Adaptive human-robot interaction control for robots driven by series elastic actuators," IEEE Transactions on Robotics, vol. 33, no. 1, pp. 169-182, 2017.

[52] M. Bertoluzzo, G. Buja, and R. Menis, "Direct torque control of an induction motor using a single current sensor," IEEE Transactions on Industrial Electronics, vol. 53, no. 3, pp. 778-784, 2006.

[53] M. E. Haque, L. Zhong, and M. F. Rahman, "A sensorless initial rotor position estimation scheme for a direct torque controlled interior permanent magnet synchronous motor drive," IEEE Transactions on Power Electronics, vol. 18, no. 6, pp. 1376-1383, 2003. 
[54] J. Maes and J. A. Melkebeek, "Speed-sensorless direct torque control of induction motors using an adaptive flux observer," IEEE Transactions on Industry Applications, vol. 36, no. 3, pp. 778-785, 2000.

[55] A. Polyakov and I. Chairez, "A new homogeneous quasicontinuous second order sliding mode control," in XVI Congreso Latinoamericano de Control Automático, 2014.

[56] A. Levant, "Sliding order and sliding accuracy in sliding mode control," International Journal of Control, vol. 58, no. 6, pp. 12471263, 1993.

[57] J. A. Moreno and M. Osorio, "Strict lyapunov funtions for the super-twisting algorithm," IEEE Transactions on Automatic Control, vol. 57, no. 4, pp. 1035-1040, 2012.

[58] L. A. Castaneda, A. Luviano-Juarez, and I. Chairez, "Robust trajectory tracking of a delta robot through adaptive active disturbance rejection control," IEEE Transactions on Control Systems Technology, vol. 23, no. 4, pp. 1387-1398, 2015.

[59] N. Martínez-Fonseca, L. Á. Castañeda, A. Uranga, A. LuvianoJuárez, and I. Chairez, "Robust disturbance rejection control of a biped robotic system using high-order extended state observer," ISA Transactions, vol. 62, pp. 276-286, 2016. 


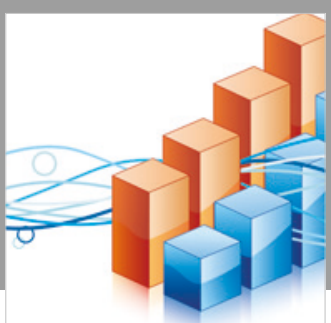

Advances in

Operations Research

\section{-n-m}
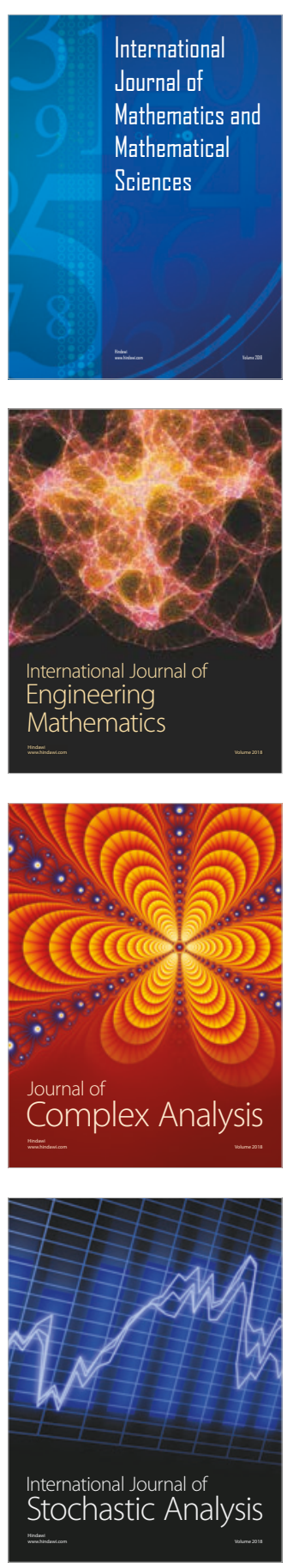
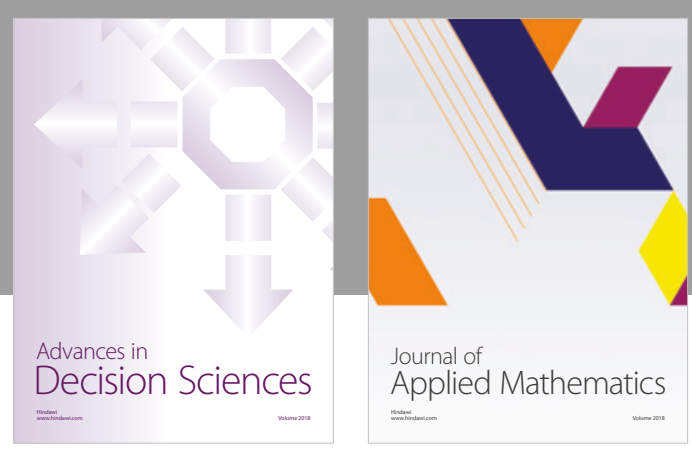

Journal of

Applied Mathematics
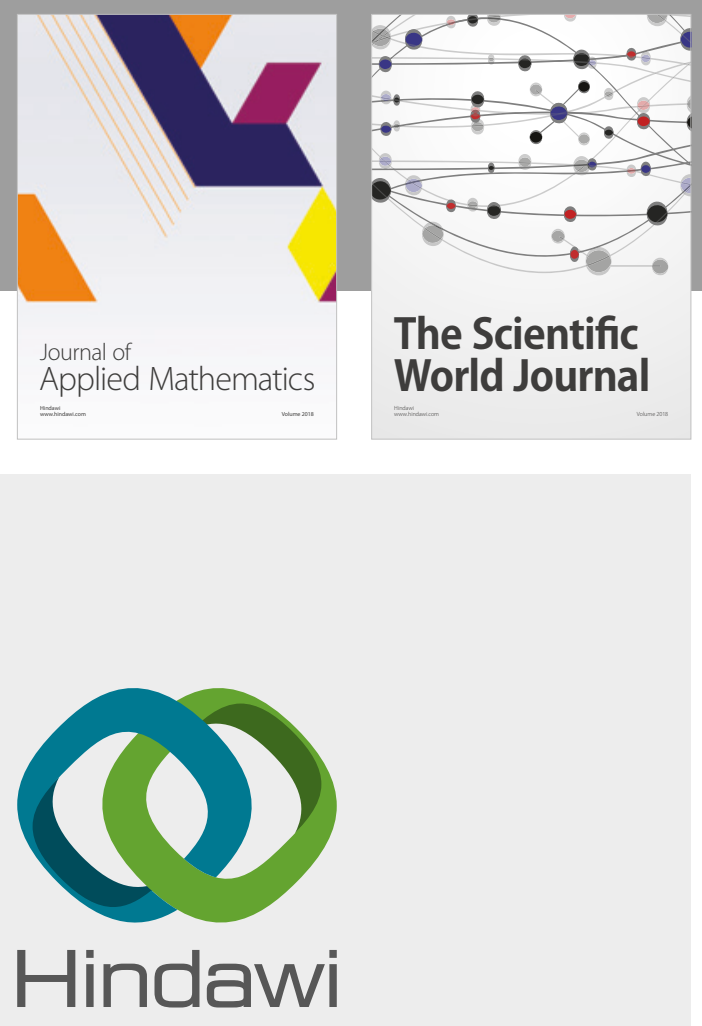

Submit your manuscripts at

www.hindawi.com

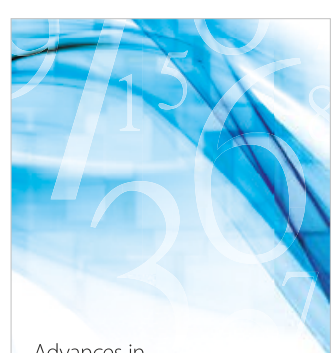

Advances in
Numerical Analysis
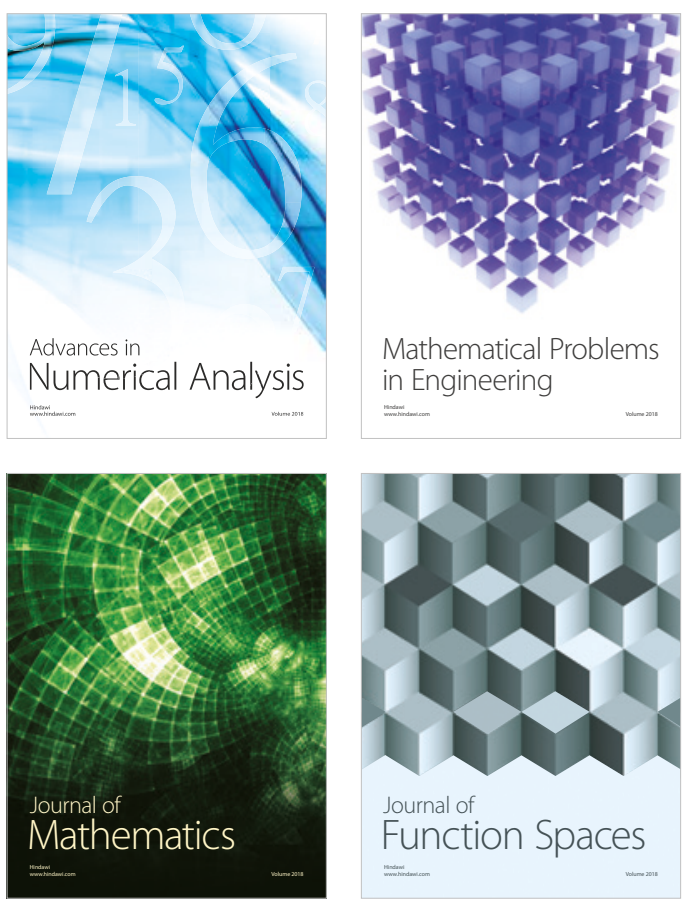

Mathematical Problems in Engineering

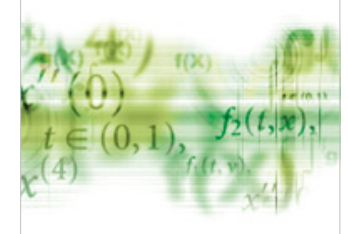

International Journal of

Differential Equations

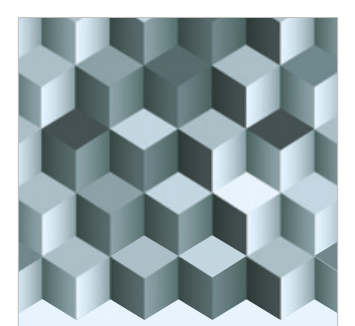

Journal of

Function Spaces

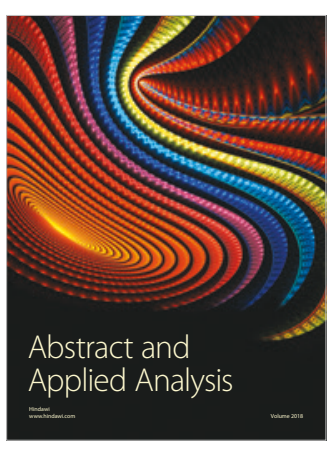

The Scientific

World Journal

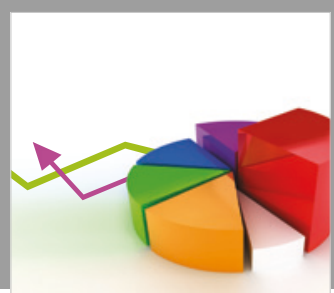

Journal of

Probability and Statistics
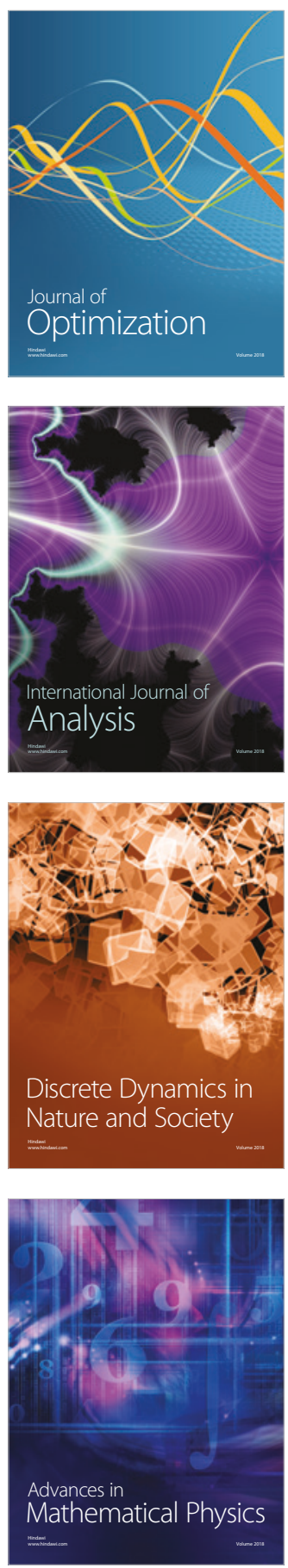